\title{
Solution-Processed Small-Molecule OLED Luminaire for Interior Illumination
}

DOE Award Number: DE-EE0001269

\section{Final Technical Report}

DuPont Displays, Inc.

Date: May 2012

Project Director:

Dr. Ian Parker

DuPont Displays, Inc.

6780 Cortona Drive

Santa Barbara, CA 93117, USA

Tel: (805) 562-5306

e-mail: lan.D.Parker@usa.dupont.com 


\section{DISCLAIMER}

This report was prepared as an account of work sponsored by an agency of the United States Government. Neither the United States Government nor any agency thereof, nor any of their employees, makes any warranty, express or implied, or assumes any legal liability or responsibility for the accuracy, completeness, or usefulness of any information, apparatus, product, or process disclosed, or represents that its use would not infringe privately owned rights. Reference herein to any specific commercial product, process, or service by trade name, trademark, manufacturer, or otherwise does not necessarily constitute or imply its endorsement, recommendation, or favoring by the United States Government or any agency thereof. The views and opinions of authors expressed herein do not necessarily state or reflect those of the United States Government or any agency thereof. 


\section{TABLE OF CONTENTS}

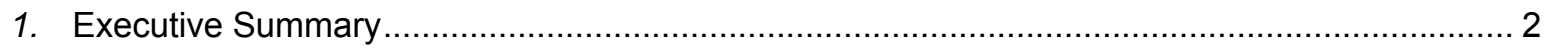

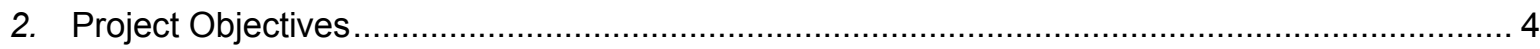

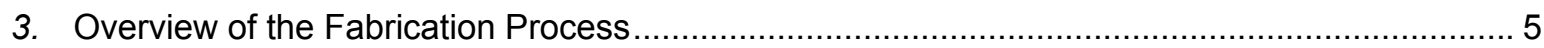

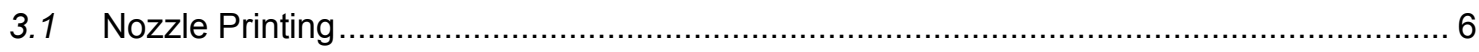

4. OLED Lighting Panels vs. AMOLED Displays................................................................ 7



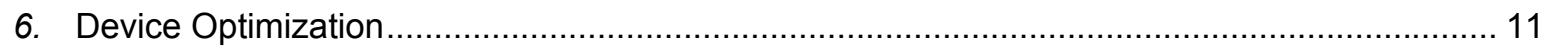

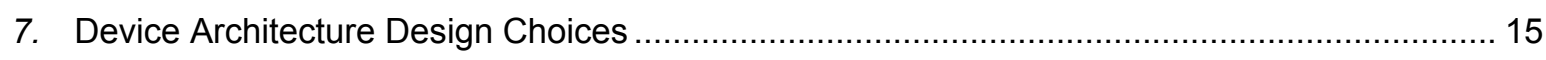

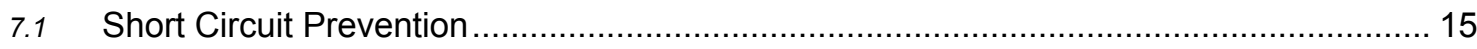

7.2 Thick Layer for Anti-Shorting Properties.................................................................... 17

7.3 Use of an n-Doped Electron Transport Layer to Lower the Operating Voltage ................ 20

7.4 Outcoupling Enhancement Films.............................................................................. 21

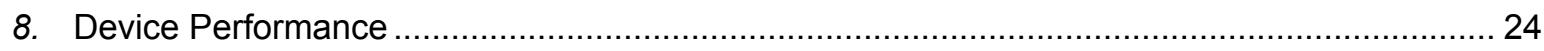

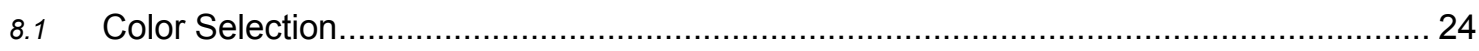

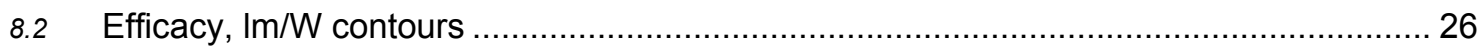

8.3 Discussion of Limited Blue Performance ............................................................... 26

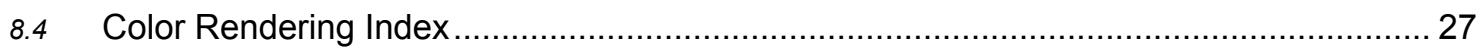

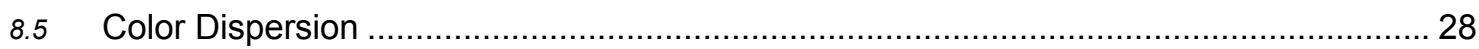

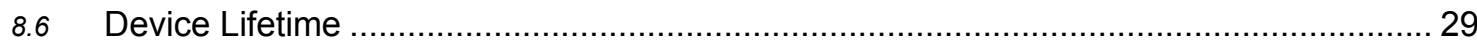

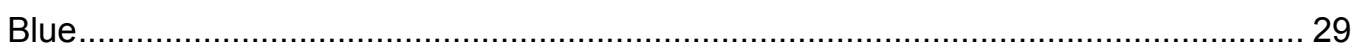

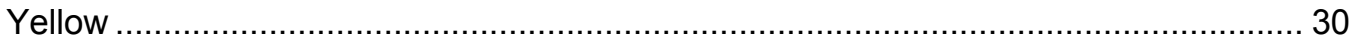

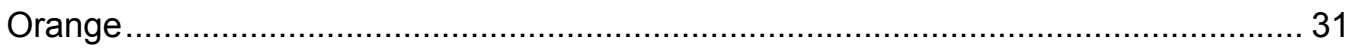

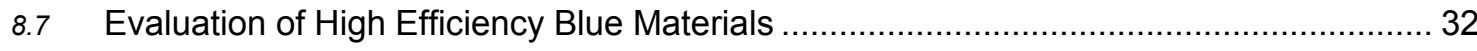



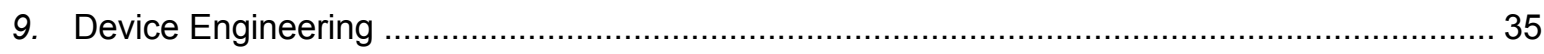

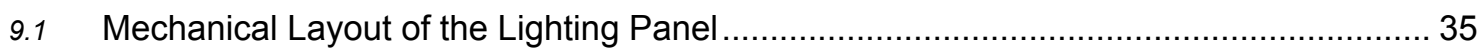

9.2 Thermal Characterization of the Lighting Panel ......................................................... 36

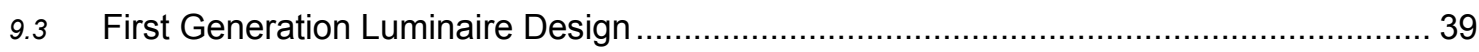

9.4 Thermal Management and Panel Redesign ....................................................... 40

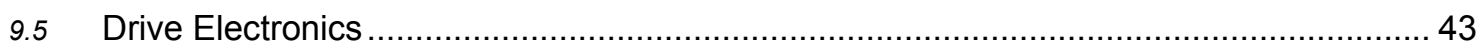

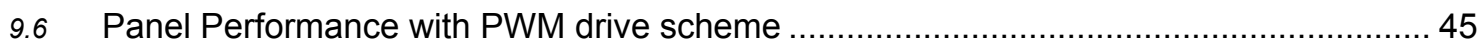

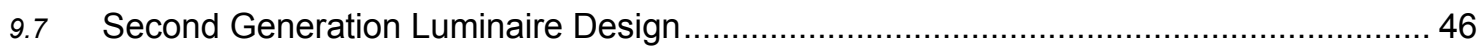

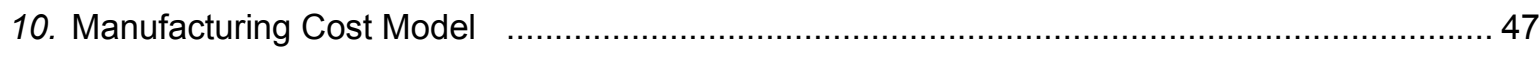



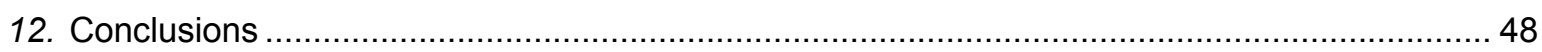

Appendix A CONFIDENTIAL

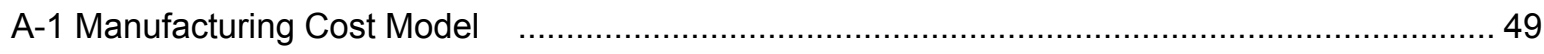

A-2 Comparison of Cost Model with DOE's Multi Year Program Plan............................................ 55 


\section{Executive Summary}

Prototype lighting panels and luminaires were fabricated using DuPont Displays' solutionprocessed small-molecule OLED technology. These lighting panels were based on a spatiallypatterned, 3-color design, similar in concept to an OLED display panel, with materials chosen to maximize device efficacy. The majority of the processing steps take place in air (rather than high vacuum). Optimization of device architecture, processing and construction was undertaken, with a final prototype design of $50 \mathrm{~cm}^{2}$ being fabricated and tested. Performance of these panels reached $35 \mathrm{Im} / \mathrm{W}$ at illuminant-A. A unique feature of this technology is the ability to color tune the emission, and color temperatures ranging from 2700 to $>6,500 \mathrm{~K}$ were attained in the final build. Significant attention was paid to low-cost fabrication techniques. 


\section{Project Objectives}

DuPont Displays, Inc. (DDI) has considerable expertise in the research and development of AMOLED displays, having developed a solution-processed small-molecule OLED technology. This technology is now well advanced and moving into the manufacturing phase, with a recent technical licensing agreement (to a large-scale display manufacturer), substantial capital investments in facilities for the manufacturing-scale production of OLED, and the joint development of manufacturing-scale nozzle-printing equipment with a major equipment vendor.

The objective of this program was to establish if this same technology (with only minor variations) could be used for the fabrication of lighting panels. Particular emphasis was placed on being able to fabricate high-efficacy luminaires with a low manufacturing cost, and with unique color-tunability properties. It should be noted that, unlike AMOLED displays, these lighting panels have No TFT backplane

Technically, the aim was to develop luminaires with efficiencies over $40 \mathrm{Im} / \mathrm{W}$ at $1000 \mathrm{~cd} / \mathrm{m}^{2}$, $\mathrm{T}_{70}>5000$ hours and a color rendering index $>85$, suitable for an interior general illumination application.

The project also aimed to demonstrate strategies for the cost-effective production of OLED lighting panels. Careful modeling suggests that DDI's manufacturing process is likely to be low cost due to the simplicity of the processes (for example, the majority of process steps take place in air rather than high vacuum), the use of lower cost equipment, the high-efficiency materials usage, the ability to scale to large motherglass size and the lowering of maintenance costs. We use a combination of slot-die coating and nozzle-printing to deposit the majority of the organic layers of the lighting panel, considerably simplifying the manufacturing equipment and allowing a low manufacturing TAC time. This cost-model is presented in Section 10 which justifies these claims. Eventually, solution processing of OLEDs for lighting may lead to low cost roll-to-roll processing, however, this was out of scope for this current project.
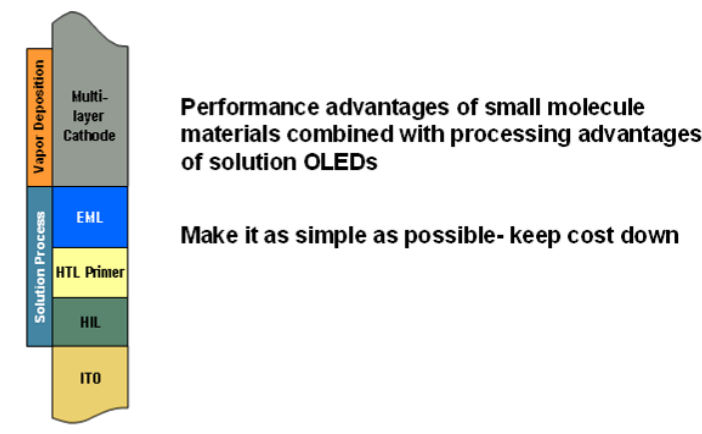

Performance advantages of small molecule materials combined with processing advantages of solution OLEDS

Make it as simple as possible- keep cost down

Fig.1. Keep manufacturing costs as low as possible with a simple design.

This project leveraged the resources of DuPont's large OLED materials synthesis team and extensive library of existing materials to select high performance small molecule emissive materials suitable for SSL. Some of these materials (e.g. the HIL, HTL and ETL materials) were materials that have proven successful for the displays program, but the emissive layers were specific to the lighting program (as discussed in detail in section 8). Although materials synthesis was not a part of this program, DDl's chemists were able to provide emissive materials that were well suited to the needs of this project. 


\section{Overview of the Fabrication Process}

DuPont Displays is taking a fundamentally different approach to OLED lighting panel fabrication. Processing of the organic layers is from solution, using slot-coating and nozzle-printing processes. These have been demonstrated to be high-yield, low TAC time processes. Furthermore, fabrication takes place in air under regular clean-room conditions, rather than requiring high-vacuum processing.

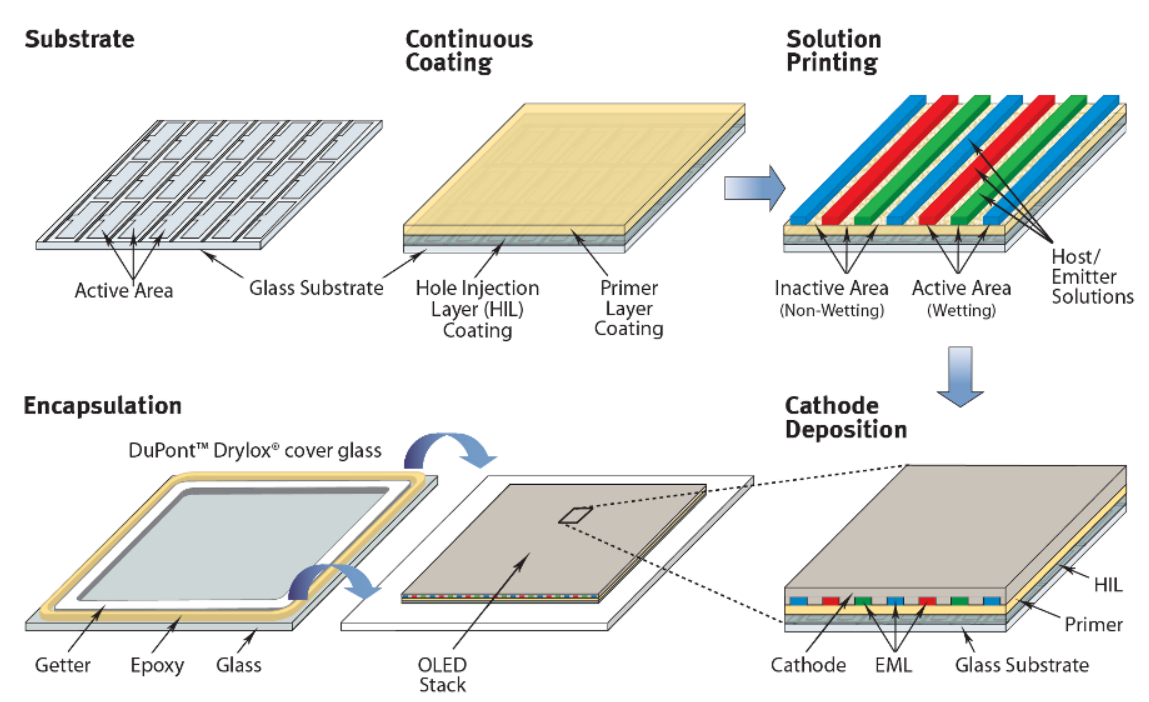

Fig.2. Process Flow for DDI's OLED lighting panel fabrication process.

Lighting panels start with a glass substrate with patterned ITO and metal bus lines. Substrates also include containment structures to define the pixels which are patterned using standard photolithography techniques. Slot-die coating is used to coat the hole-injection layer (HIL) from aqueous solution, followed by a second pass through a slot-die coater for the hole-transport I primer Layer (HTL). These layers are common to all pixels and consequently are not patterned. The light-emitting layers (EML) are patterned using nozzle-printing equipment (see Section 3.1). Pixels are printed in columns with lateral dimensions of $154 \mu \mathrm{m}$. Both the slot-die process steps and the nozzle-printing are carried out in a standard clean-room atmosphere. The device is transported to a vacuum chamber only for the electron-transport layer (ETL) and metal cathode deposition. After cathode deposition, the whole luminaire is hermetically sealed using a glass lid containing DuPont ${ }^{\mathrm{TM}}$ Drylox ${ }^{\circledR}$ technology (a low-cost, screen-printed desiccant), trapping dry $\mathrm{N}_{2}$ gas between the lid and cathode.

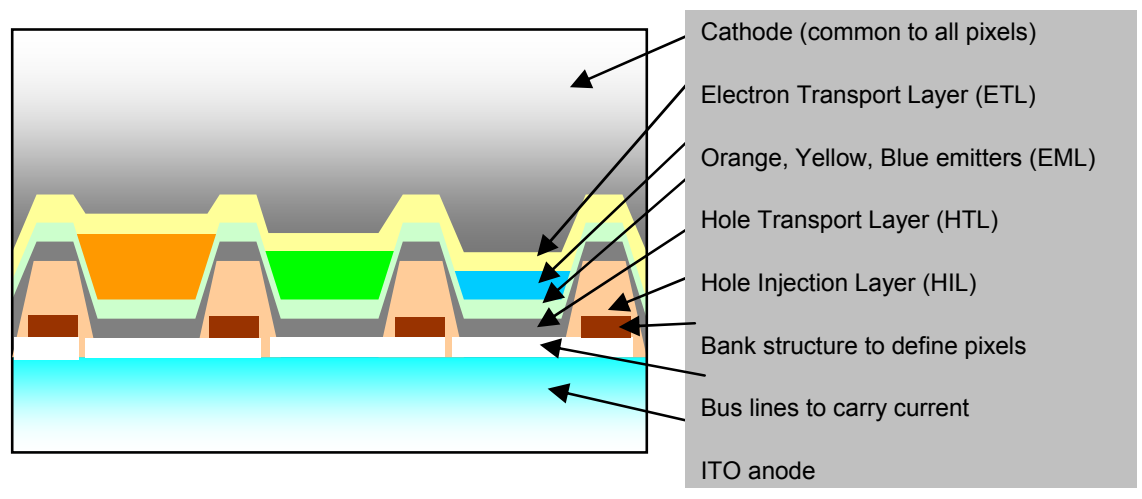

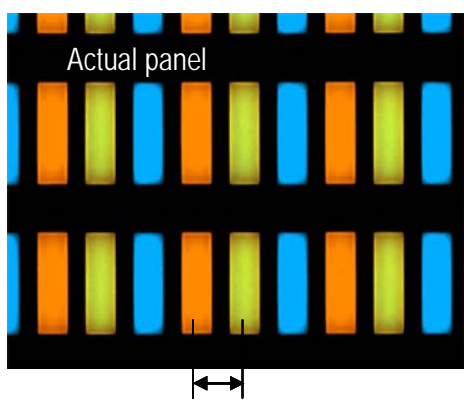

$154 \mu \mathrm{m}$

Fig.3. Cross-section through the panel, and a photograph showing pixel arrangement 
Fig. 3 shows the cross-section through the lighting panel. This is similar to an AMOLED except for the lack of TFT structure for each pixel, replaced instead by the current bus lines connecting the whole column.

\subsection{Nozzle Printing}

In the EML deposition step, solutions of organic materials are 'nozzle printed' i.e. continuously jetted through an array of nozzles moving at high speed. This equipment has been developed, in conjunction with the Japanese semiconductor equipment manufacturer SCREEN, (formerly known as Dainippon Screen Mfg. Co., Ltd.) and is a Gen4 tool designed for high throughput.

In this printer, the print head moves across the substrate at a speed of $5 \mathrm{~m}^{2}$, and is able to print 15 columns of the panel simultaneously (see Fig.4). The TAC time for a manufacturing line containing a single Gen4-sized printer, printing onto substrates with 6 lighting panels (each having an emissive area of $0.1 \mathrm{~m}^{2}$ ) will be 1 minute per substrate, corresponding to a throughput of $37 \mathrm{k}$ substrates/month (or $220 \mathrm{k}$ lighting panels/month) - see the cost model in section 10 for more details.

Simultaneous jetting from 15 nozzles presents a technical challenge since each nozzle acts as an independent flow element with a separate mass flow controller. As a consequence, short range luminance variations can occur unless the nozzle flows are tightly matched - a consequence of differences in the film thickness. In practice flow rates are highly consistent, producing films with thickness differences of less than $1 \%$. In terms of short-range luminance uniformity (an industry metric for AMOLED displays), display panels produced by DDI, using our Gen4 nozzle printer, outperformed commercial AMLCD displays, demonstrating the high uniformity that can be achieved using nozzle printing technology. (See "Measurement Methods for Solution-Coated AMOLED Display Uniformity", SID 2011 Digest, P-78, J.Ziebarth, A.Andreatta, R.Chesterfield, K.Frischknecht, A.Johnson, and M.Stainer: DuPont Displays, Santa Barbara, CA USA).
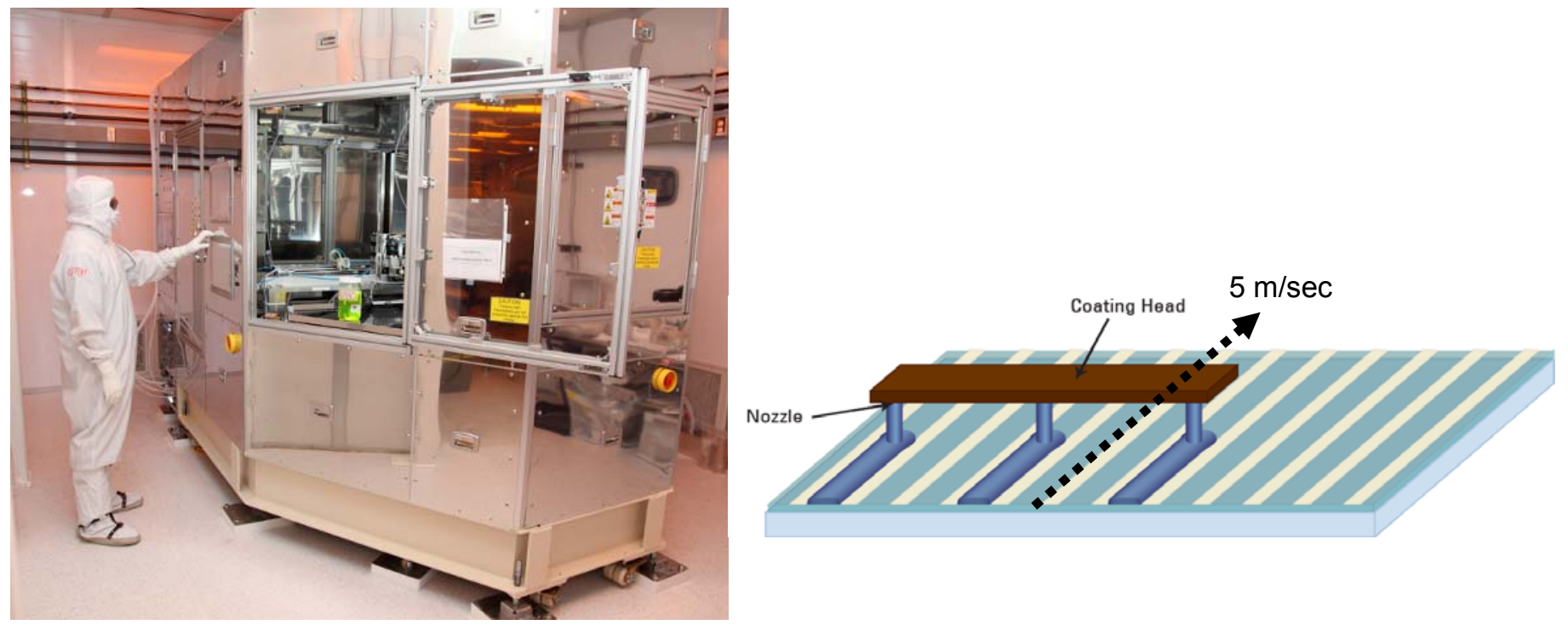

Fig.4. DDI's manufacturing scale printer from SCREEN, capable of printing Gen4 panel (glass sheets up to $920 \times 730 \mathrm{~mm}$ ) with high throughput. The footprint is approx. $2 \mathrm{~m} \times 1.5 \mathrm{~m}$. 


\section{OLED Lighting Panels vs. AMOLED Displays}

For displays we choose materials that give us a large color-gamut in order to best represent video images. For lighting we don't need this wide range of colors. We select our materials based on highest efficacy instead.
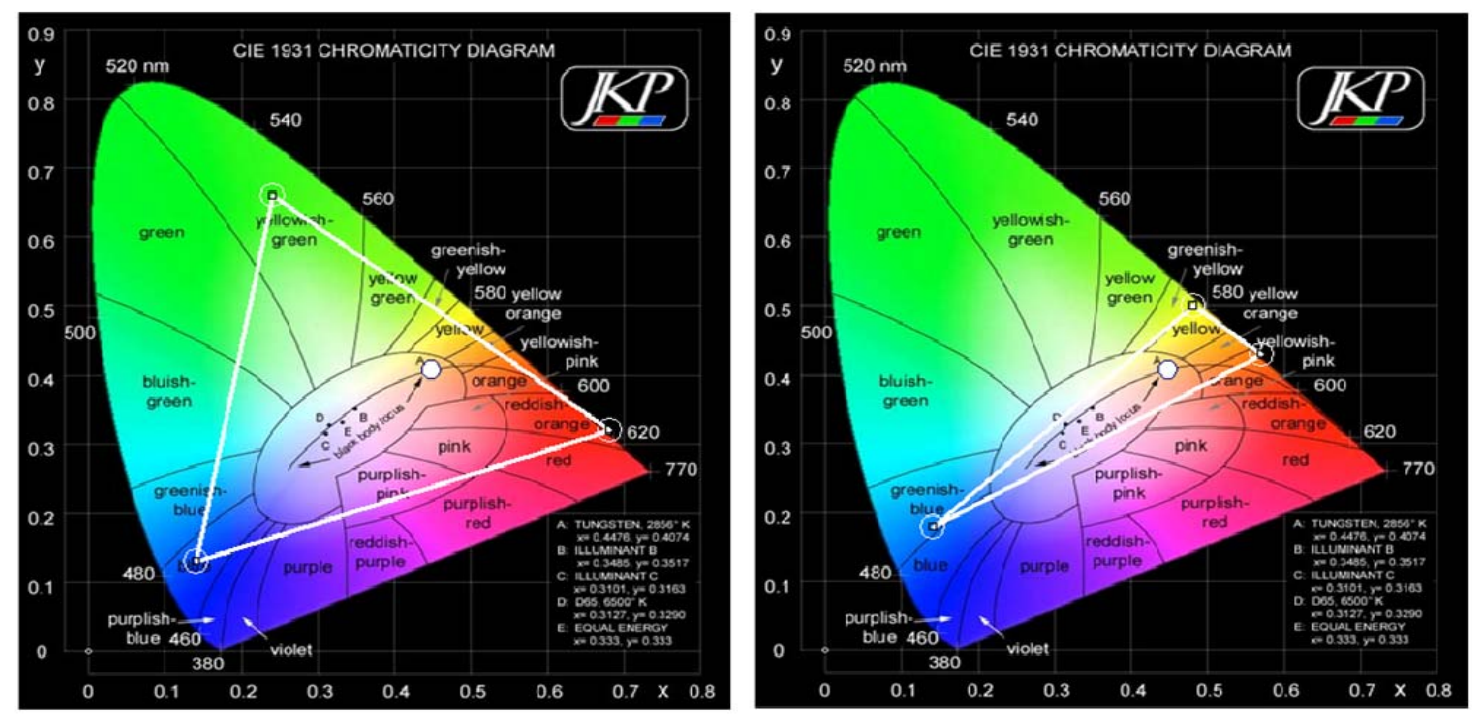

Fig.5. The image on the left shows the typical color gamut for an AMOLED display. Compare this to the reduced color gamut on the right, for a lighting panel

In general, the use of high efficiency materials is desirable since their EQE values are generally much higher than fluorescent materials - this can be seen in Table 6. In addition, deep red and deep blue colors are less desirable due to the poor eye sensitivity to these colors. Hence, a high efficiency orange is a better choice than a high efficiency red material. Such an orange material is better coupled with a yellow material rather than a deep green since forming a white from orange+green requires a disproportionately large orange contribution that will adversely affect overall efficacy.

To demonstrate this, Fig. 6 shows a comparison between and red-green-blue (RGB) set of display materials (with a gamut of $66 \%$ of NTSC) compared with a reduced gamut set of lighting materials chosen based on their efficacy (gamut is only $15 \%$ of NTSC). As expected the combined 'white' performance is considerably higher for the lighting set.

It will be noted that the blue material is a fluorescent emitter in both cases (in fact it is the same emitter but the optical cavity has been adjusted to give different colors). Currently available high efficiency blue materials generally are too green to be used in the color-mixing manner that is required here. Also, their operational stability is poor. The lack of a viable high efficiency blue material is a limitation which currently impacts the achievable performance of the lighting panels. This is discussed in more detail in sections 8.6 and 8.7. 
It should be noted that, unlike for a display, a wide range of possible colors is acceptable for the primaries of a lighting device, since the final panel has a tunable color. This gives considerable latitude in the choice of material.

\begin{tabular}{|l|c|c|c|c|c|}
\hline \multicolumn{2}{|l|}{ DISPLAY COLORS } & \multicolumn{3}{|c|}{ Measured with integrating sphere } & $\begin{array}{c}\text { Photometer @ } \\
1,000 \mathrm{~cd} / \mathrm{m} 2\end{array}$ \\
\hline & CIE & $\begin{array}{c}\text { Meas. } \\
\text { condition }\end{array}$ & $\begin{array}{c}\text { Lm/W } \\
\text { with OE }\end{array}$ & $\begin{array}{c}\text { EQE (\%) } \\
\text { with OE }\end{array}$ & $\begin{array}{c}\text { cd/A } \\
\text { with OE }\end{array}$ \\
\hline $\begin{array}{l}\text { Deep red } \\
\text { RD891 }\end{array}$ & $(0.65,0.35)$ & $2100 \mathrm{Im} / \mathrm{m}^{2}$ & 10.3 & 16.8 & 22.1 \\
\hline $\begin{array}{l}\text { Green } \\
\text { G1973 }\end{array}$ & $(0.27,0.63)$ & $2600 \mathrm{Im} / \mathrm{m}^{2}$ & 11.5 & 5.8 & 23 \\
\hline $\begin{array}{l}\text { Deep Blue } \\
\text { BD1900 }\end{array}$ & $(0.14,0.14)$ & $1800 \mathrm{Im} / \mathrm{m}^{2}$ & 3.0 & 4.3 & 6.2 \\
\hline
\end{tabular}

These give a combined performance of $8 \mathrm{Im} / \mathrm{W} @ 1,000$ nits

for white (illuminant. A)

\begin{tabular}{|l|c|c|c|c|c|}
\hline \multicolumn{2}{|l|}{ LIGHTING COLORS } & \multicolumn{3}{|c|}{ Measured with integrating sphere } & $\begin{array}{c}\text { Photometer @ } \\
1,000 \mathrm{~cd} / \mathrm{m} 2\end{array}$ \\
\hline CIE & $\begin{array}{c}\text { Meas. } \\
\text { condition }\end{array}$ & $\begin{array}{c}\text { Lm/ } \\
\text { with OE }\end{array}$ & $\begin{array}{c}\text { EQE (\%) } \\
\text { with OE }\end{array}$ & $\begin{array}{c}\mathrm{cd} / \mathrm{A} \\
\text { with OE }\end{array}$ \\
\hline $\begin{array}{l}\text { Orange } \\
\text { R2640 }\end{array}$ & $(0.56,0.44)$ & $2100 \mathrm{Im} / \mathrm{m}^{2}$ & 30 & 21.6 & 55 \\
\hline $\begin{array}{l}\text { Yellow } \\
\text { Y2392 }\end{array}$ & $(0.47,0.52)$ & $2600 \mathrm{Im} / \mathrm{m}^{2}$ & 45 & 21.2 & 78 \\
\hline $\begin{array}{l}\text { Pale Blue } \\
\text { BD1900 }\end{array}$ & $(0.14,0.17)$ & $1800 \mathrm{Im} / \mathrm{m}^{2}$ & 4.3 & 6.7 & 10 \\
\hline
\end{tabular}

These give a combined performance of 21 Im/W@1,000 nits

for white (illuminant. A)

Table 6. Device data from printed $2 \mathrm{~cm}^{2}$ devices. It should be noted that these data were collected at an early stage in the project. Performance of the O-Y-B lighting set was considerably improved by later optimization of the process - as described in later sections. 


\section{Device Modeling}

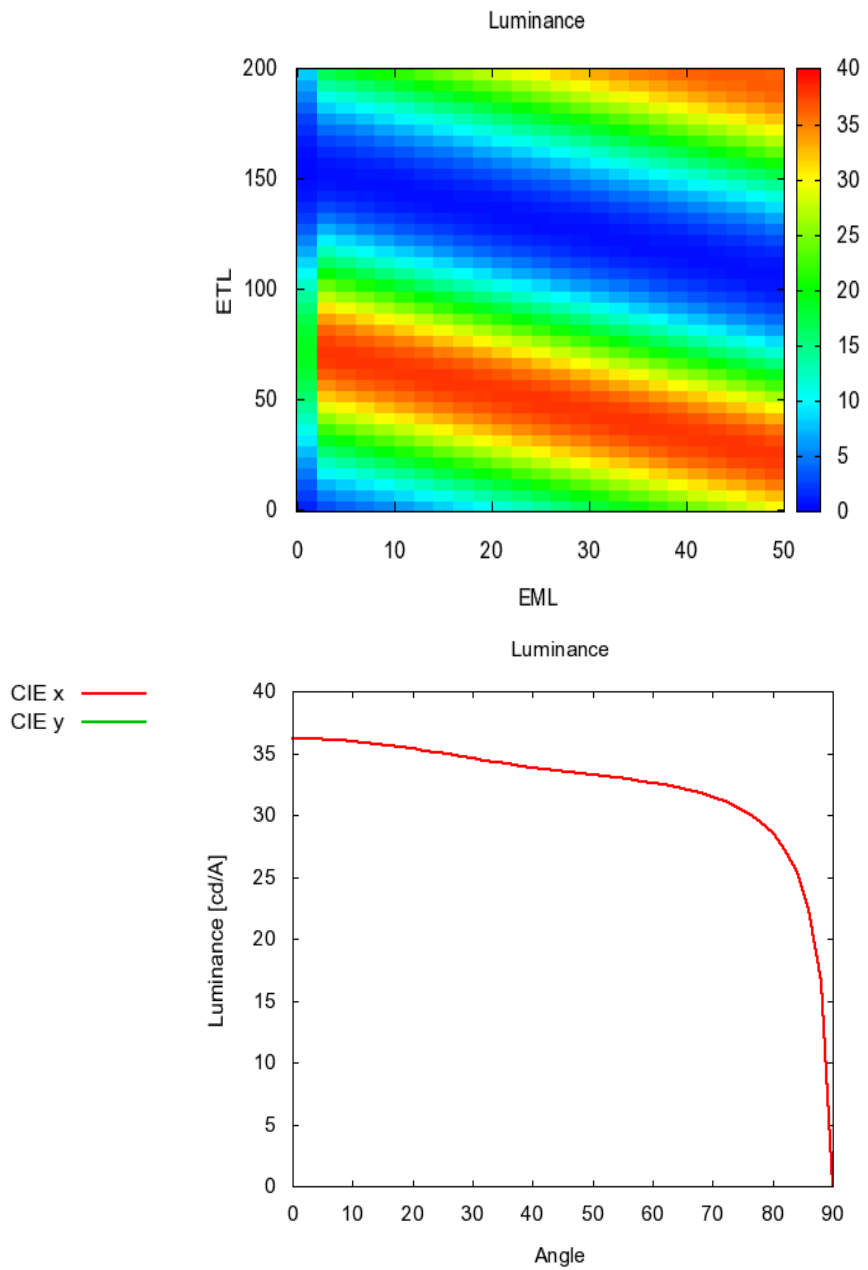

Luminance

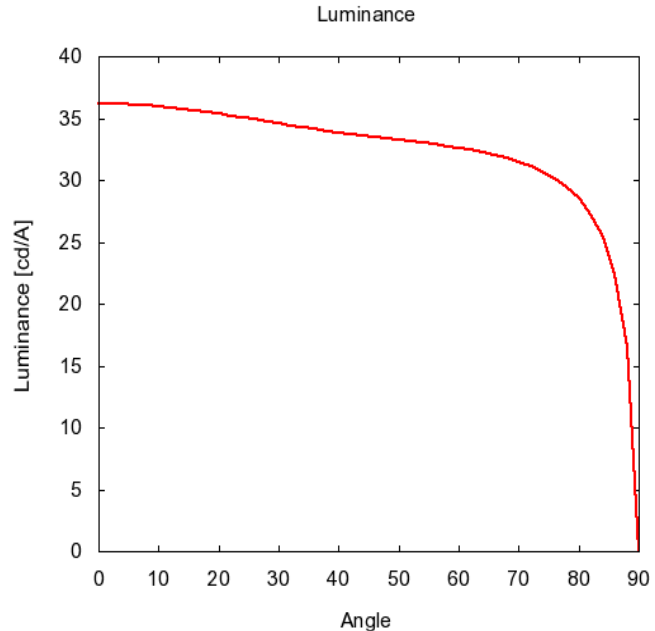

Fig.7. Examples of optical characteristics modeling from SETFOS.

During the preliminary stages of this project it was necessary to screen materials and to select an optimal device architecture. To assist this process, and to accelerate material selection, two modeling packages were used.

The commercially available SETFOS optical modeling package (Fluxim AG, Switzerland) was used to model the optical characteristics of individual colors. Since OLEDs are weak optical cavity devices, their emission characteristics are dependent on the thicknesses of the films in the multilayer structure. Using SETFOS, the optical emission characteristics could be calculated for a range of different device structures, with the aim of maximizing luminance and minimizing angular dispersion of a device. For example, the upper panels of Fig. 7 show luminance and color variations for various combinations of EML and ETL thicknesses. The periodic nature of the luminance, arising from the optical interference of the optical cavity, can be clearly seen hence the need to choose appropriate film thickness with care. The lower panels show calculations of the angular dispersion characteristics. Using this model allowed us to rapidly screen a range of potential materials from DDl's library of OLED materials for further experimental validation. 
SETFOS does, however, have limitations. For example it does not model the electrical characteristics of the device (at least not without extensive prior verification of materials properties - which were not available for all of the materials here). Consequently, important parameters such as operating-voltage and $\mathrm{Lm} / \mathrm{W}$ could not be directly modeled. Furthermore, SETFOS is not designed to model the behavior of 3-color devices, and so color-mixing and color-balance for different white-points required a different model.

Such a model was developed in house. Taking as its inputs actual device characteristics from photometer and integrating-sphere measurements $\left(\mathrm{V}, \mathrm{cd} / \mathrm{m}^{2}, \mathrm{~A} / \mathrm{m}^{2}, \mathrm{CIE}\right.$ coordinates and $\left.\mathrm{Im} / \mathrm{W}\right)$, and combining these with the user-specified target-luminance and target-color (e.g. illuminant-A or D65), the model calculated the appropriate mix of the three colors. It estimated the overall panel power $(\mathrm{W})$, current requirements and $\mathrm{Im} / \mathrm{W}$ value, along with the breakdown of these values for the individual colors. This model proved to be very useful for materials selection and estimation of panel performance.

An example for an orange-yellow-blue combination is shown in fig. 8. In this case the individual O-Y-B device performance (from actual test coupons) is shown (1), along with the measured CIE coordinates (2). The target color and luminance are provided (3). The model then calculates the mix of the three colors required to give the target color and luminance (4), from which the operating-point for each color is calculated (5), and then the lumens and power for each color (6). Finally the overall $\mathrm{Im} / \mathrm{W}$ and power for the panel is calculated (7), based on the panel dimensions.

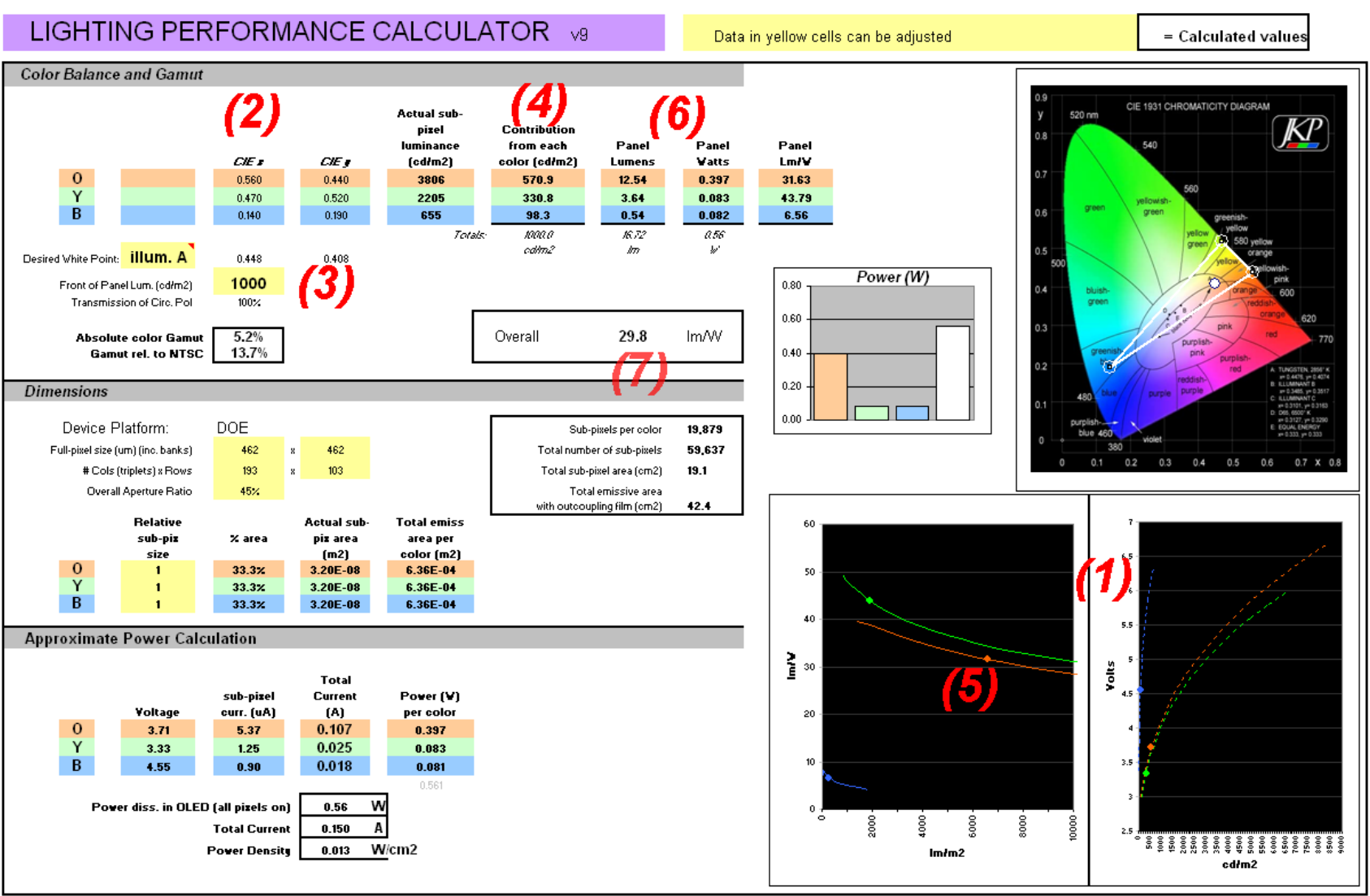

Fig.8. Model developed to assess the overall performance for a lighting panel, given the desired panel luminance and color-point. Actual device data (from the integrating sphere) is used to predict the performance at the working point. 


\section{Device Optimization}

Small Test Coupons: Initial screening work for new materials and new process conditions was handled with small-area devices - single pixels with an area $\sim 5 \mathrm{~mm}^{2}$, fabricated by spin-coating techniques (not printing), and with device fabrication taking place in a glove-box. This was for practical expediency (rapid turnaround of data) and for historical consistency. These devices were tested using calibrated equipment. PhotoResearch-750 photometers were used for color, forward luminance and angular dependence. A calibrated integrating sphere (8") was used to measure lumens and Im/W. And a Keithley-2400 source-measure unit was used to measure for the current-voltage curves.

$\mathbf{2} \mathbf{c m}^{2}$ Printed Test Coupons: Chosen materials were then evaluated with $2 \mathrm{~cm}^{2}$ active area test coupons, which were printed in the manner described in section 3 . These devices were monochromatic, with all pixels ganged together. These devices were printed on 6" 6 " glass substrates, each substrate containing 16 devices (see fig. 9 below). Individual devices were scribed and cut from these panels and tested using calibrated equipment. PhotoResearch-750 photometers were used for color, forward luminance and angular dependence. A NISTtraceable integrating sphere system (LabSphere, 20" diameter halfmoon system with CDS-1100 spectrometer) was used to measure lumens and Im/W. And a Keithley-2400 source-measure unit was used to measure for the current-voltage curves. Device data was analyzed statistically using MINITAB software. Typical device data is shown in Figs.10 and 11. Reference to "diffuser" in these figures relates to outcoupling enhancement films that are discussed in detail in section 7.4 .

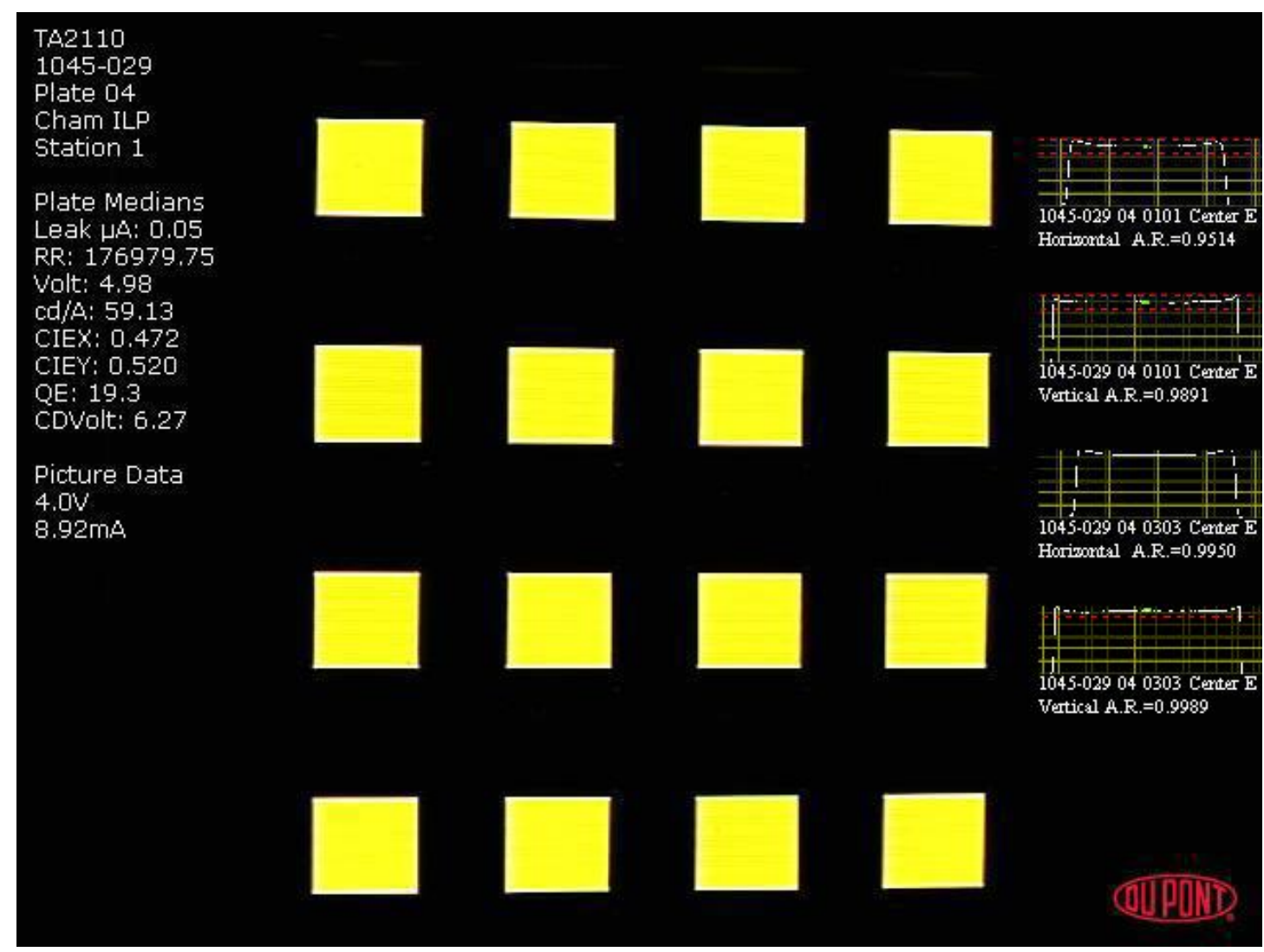

Fig.9. An example of the initial testing for a 6"x6" plate containing sixteen, $2 \mathrm{~cm}^{2}$ yellow, printed devices. 

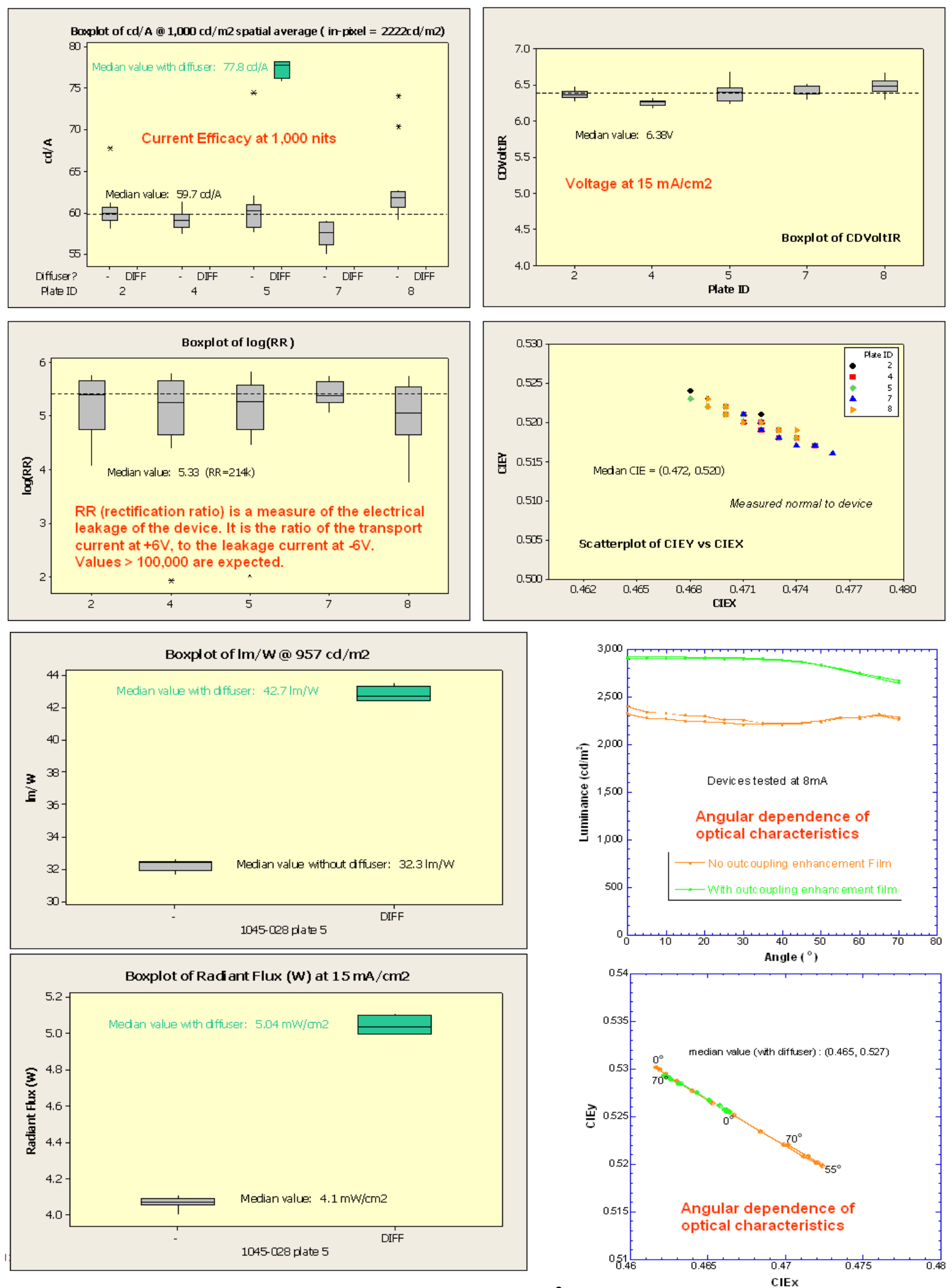

Fig.10. An example of typical device performance for $2 \mathrm{~cm}^{2}$-sized printed yellow devices. The sample size is 80 devices from five, individually printed 6x6 plates (each with 16 devices). These data were collected early in the project and subsequent optimization of the devices increased their performance. Reference to "diffuser" in these figures relates to outcoupling enhancement films that are discussed in detail in section 7.4. 


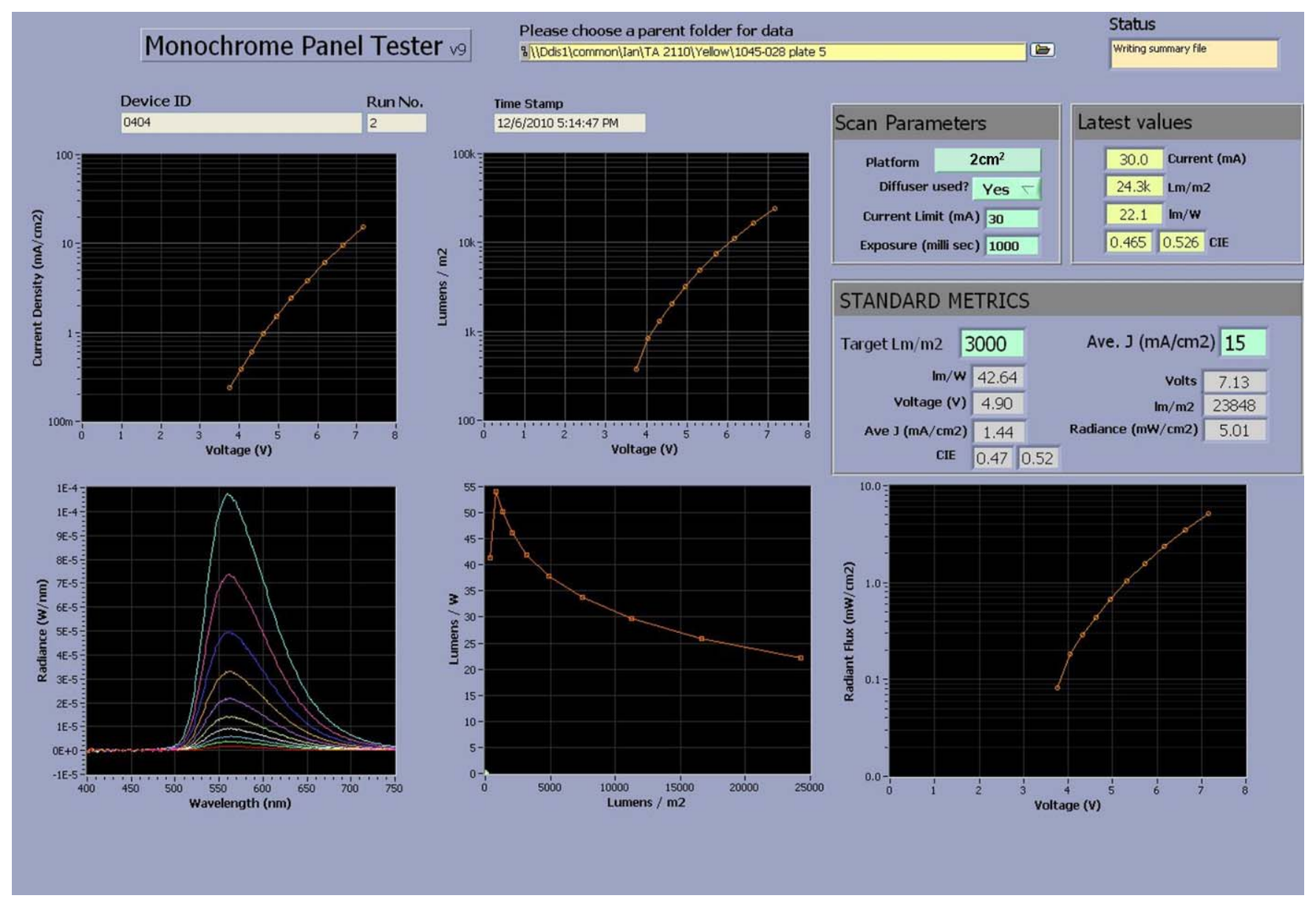

Fig.11. Typical integrating sphere performance for $2 \mathrm{~cm}^{2}$-sized printed yellow devices.

Mid-Sized Panels: After the final materials selection was completed, 3-color lighting panels were fabricated having an active area of $50 \mathrm{~cm}^{2}$. These were fabricated in the manner described in section 3. The size and pixel layout of these panels was chosen to be similar to existing AMOLED panels used in DDI's displays program, thereby minimizing the need for refixturing of the process line. One limitation caused by this choice however, was unused area on the panel area that, with an AMOLED, would be occupied with TFT circuitry (see Fig.13). In future iterations of this design, this area will be reclaimed for emissive area.

These panels were capable of being driven as color-tunable white devices. Testing of these devices was performed using a NIST-traceable integrating sphere system (LabSphere, 20" diameter halfmoon system with CDS-1100 spectrometer). Custom software was written to drive the integrating sphere: initially each color was measured individually, then using this data, the computer calculated the appropriate color balance for the chosen white-point and this whitepoint was maintained as the panel was swept through a range of emittance levels $\left(\mathrm{Im} / \mathrm{m}^{2}\right)$. 


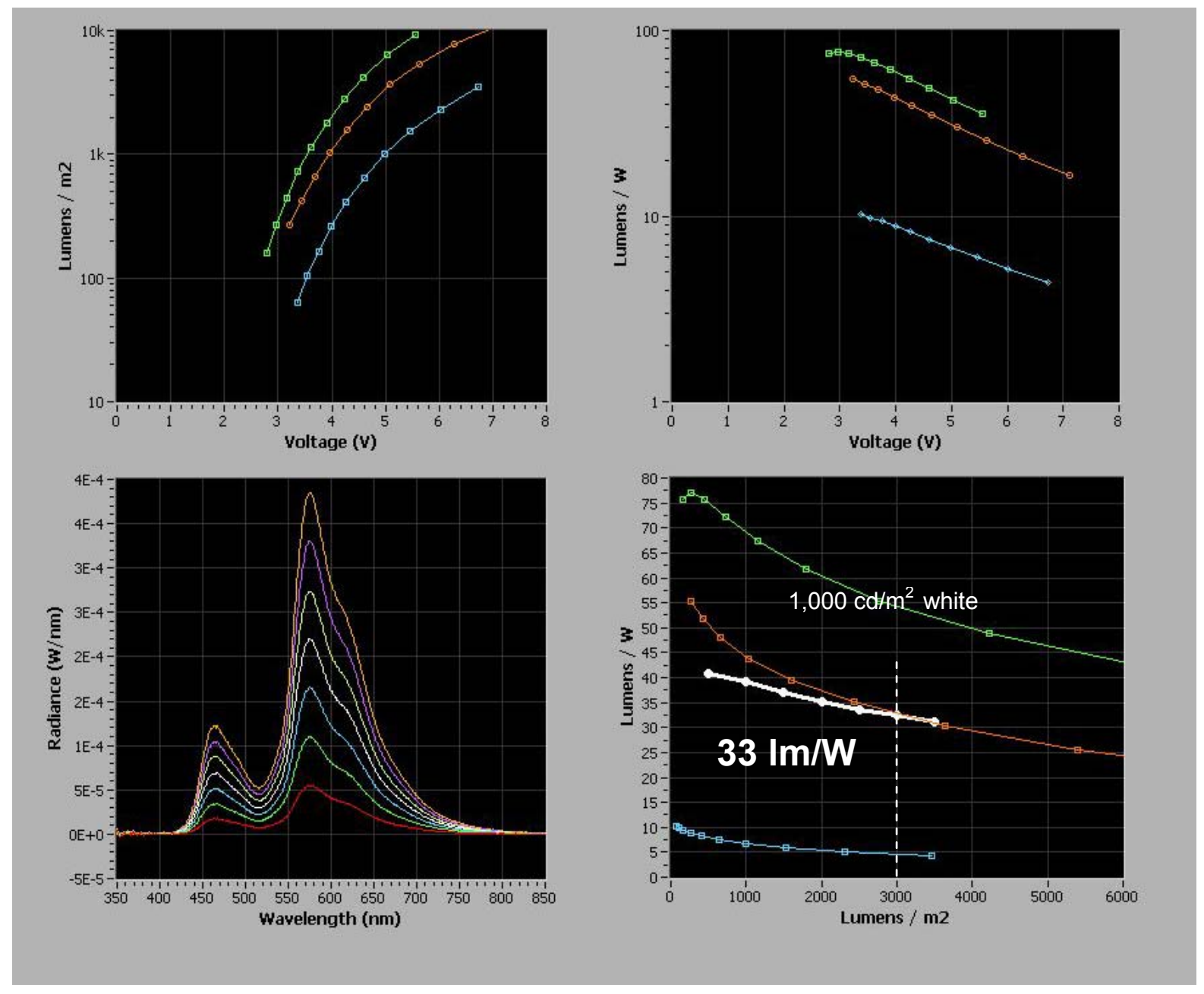

Fig.12. A typical data set for a $50 \mathrm{~cm}^{2}$ panel tested in the 20 " integrating sphere. The orange, green and blue curves indicate the performance of the three primary components. The white curve indicates the combined output, in this case tuned to illuminant-A. The spectra are for the white output. 


\section{Device Architecture Design Choices}

\subsection{Short Circuit Prevention}

In order to allow simple drive electronics (and consequently keep fabrication costs at a minimum), the $50 \mathrm{~cm}^{2}$ panels employed a significant degree of "ganging" together of electrodes. A potential problem for such a design is that an electrical short, located on a single pixel, if severe enough, could lead to the loss of one of the component colors.

To address this possibility we designed the pixels with individual "limiting resistors", so that a short in any one pixel would be isolated to that pixel - the rest of the luminaire would continue to function with an unnoticed reduction in light output. This is shown in Fig.13 - the ITO layer of the pixel is connected to the metal bus line by a 'serpentine' of ITO - sufficient to carry the current during operation with a small voltage drop, but with sufficient resistance to limit any current into the pixel if a short should develop, thereby isolating the short to that pixel. This necessitates 'pixellation' of the ITO, rather than using continuous columns of ITO, but the associated breaks in the ITO lanes can be made sufficiently small so as to minimize loss in active area. In practice, this break was larger than desired since, as previously mentioned, the panel design was based on an earlier AMOLED layout, and contained 'unused' space that would have otherwise been occupied with TFT circuitry. Future iterations of the panel will reclaim this area for emissive regions.

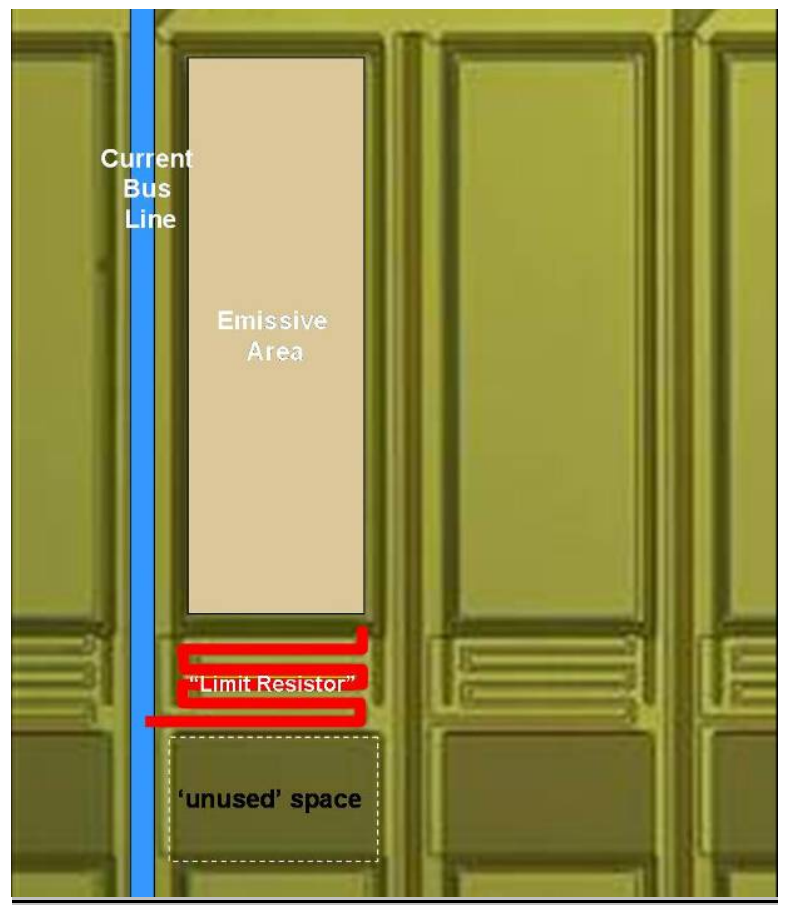

Fig.13. Structure of a pixel on the $50 \mathrm{~cm}^{2}$ panels showing the 'limiting resistor' for short-circuit prevention.

The size of the 'limiting resistor' was chosen to be $2 \mathrm{k} \Omega$ based on SPICE-modeling, as shown in Fig.14. This was large enough to limit any runaway current, but small enough to have a negligible voltage drop for normal behavior. For example: the current into an individual pixel during normal operation is $\sim 1-5 \mu \mathrm{A}$, so (at $2 \mathrm{k} \Omega$ ) the limit resistor only drops $2-10 \mathrm{mV}$, which is insignificant. However, if a severe short were to develop in the pixel, the overall resistance will not drop below the 
limit value of $2 \mathrm{k} \Omega$, and the current will be limited to $\sim 5 \mathrm{~V} / 2 \mathrm{k} \Omega \sim 2.5 \mathrm{~mA}$. Since the resistance of the entire-column current-bus is $\sim 50 \Omega$, the maximum voltage drop along the bus is $2.5 \mathrm{~mA} \times 50 \Omega \sim 125$ $\mathrm{mV}$, causing a luminance drop that is only just noticeable. This is the worst-case scenario, in most cases the voltage drop is much less.


Fig.14. (Upper panel) A SPICE model was used to simulate the effect of a shorted-pixel. The model represented a 12 pixel section of a column of the device. The simulated 'short' is represented by placing a small-value resistor in parallel with the OLED.

(Lower panel) The results of the SPICE model for different degrees of shorting $\left(R_{\text {short }}\right)$, showing the current through the shorted pixel and, more importantly, how the current through the neighboring pixels is barely affected if the limiting resistor $\left(R_{\text {limit }}\right)$ is high enough. The vertical axis represents current $(\mu A)$. 


\subsection{Thick Layer for Shorting Reduction}

A further means of reducing the likelihood of shorting within a pixel is to fabricate a device with thicker layers. This is simply a mechanical approach - thicker films are better at 'burying' particles, asperities or coating defects which might otherwise disrupt film coating and cause an electrical weak-spot.

Optical modeling was performed for candidate structures to choose the appropriate thicknesses for various layers. Since cavity effects can be strong for these structures, only certain narrow ranges of film thicknesses are appropriate. An example of such modeling (using the SETFOS package) is shown in Fig.15, the range of 'high luminance' possibilities is indicated as the $1^{\text {st }}$ and $2^{\text {nd }}$ harmonic solutions.

There were practical constraints to be considered when designing these devices. For example, thicker films of the more electrically resistive layers (e.g. the EML layer) can lead to a high operating voltage which is undesirable for achieving high $\mathrm{Im} / \mathrm{W}$. Also some layers of the device are common to all three layers (e.g. the HIL and ETL layers) due to the fabrication process (these layers are coated with a slot-coater and are unpatterned). Consequently, an appropriate thickness that simultaneously suits all three colors was required. Furthermore, at the time of this project, it was not feasible to coat thick HIL layers due to procedural limitations in the pilot-line equipment.

Taking account of these constraints, an architecture employing a 'thick' ETL layer was selected as most likely to maximize the $\mathrm{Im} / \mathrm{W}$ performance for a combined 3-color device. As an example of this approach, Fig.15 shows 2 possible solutions for Orange and Green devices - an ETL thickness of either $50 \mathrm{~nm}$ or $160 \mathrm{~nm}$ ETL gives maximum luminance for both colors (although the individual EML thicknesses are color-specific). The 160nm ETL was considered to be a viable option, since it was anticipated that excessive voltage drop would be negated with the use of a dopant - see section 7.3. However, a competing factor was that such a thick layer might add significantly to the materials cost.

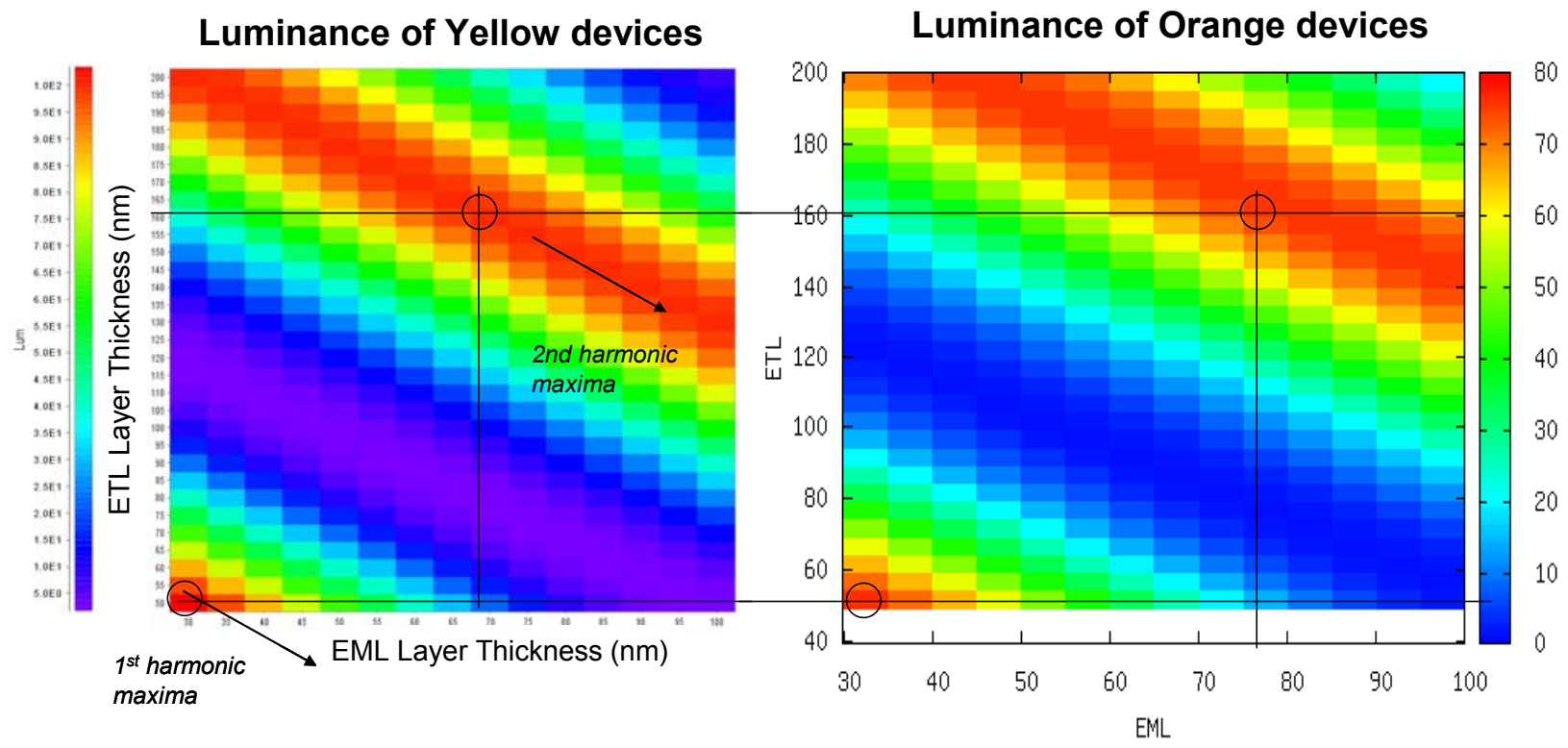

Fig.15. Modeling luminance output for different device structures using SETFOS (Fluxim AG). 
Such optical models give good guidance to performance but must be confirmed with actual device data. This was particularly true for these devices since we need to maximize the total luminance emitted in all directions - not just the forwards luminance as modeled by SETFOS.

Fig. 16 shows an example of verification data for blue devices collected using small test coupons. In this particular case, both $50 \mathrm{~nm}$ and $160 \mathrm{~nm}$ ETL devices showed local maxima in $\mathrm{Im} / \mathrm{W}$ as suggested by the model. But overall the $50 \mathrm{~nm}$ device showed a higher performance when assessed using the outcoupling enhancement film. Similar relative performance was found for orange and yellow devices also. The final decision was taken to use a 50nm ETL.

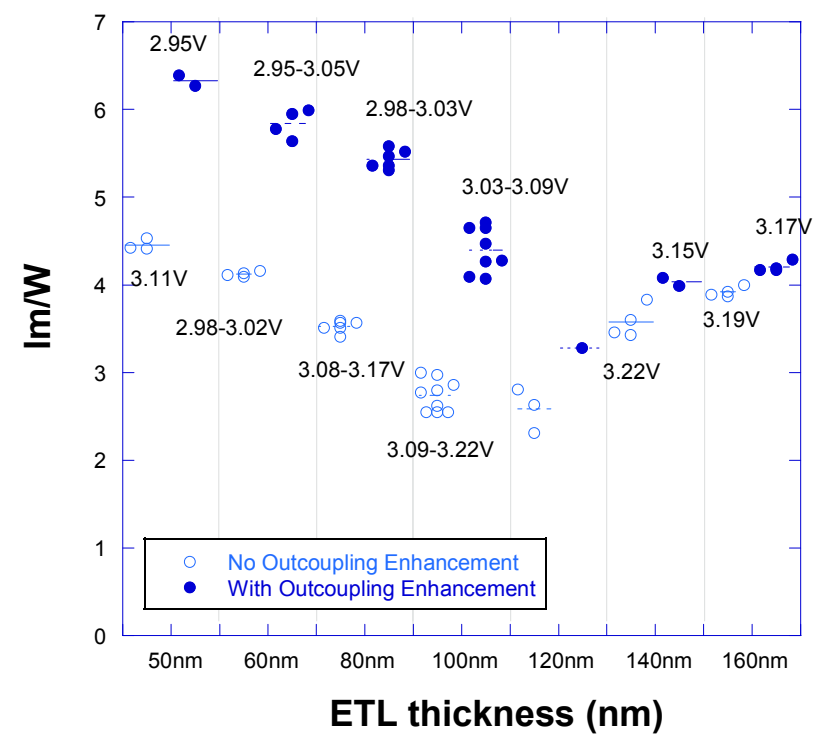

Fig.16. Data for Blue devices - both 50nm and 160nm ETL devices show local maxima in $1 \mathrm{~m} / \mathrm{W}$, but overall the $50 \mathrm{~nm}$ device has better performance when assessed with outcoupling enhancement film.

As an added demonstration that thick ETL devices can have high performance, Fig.17 shows the performance of orange devices, comparing the 'thick' 50nm ETL structure with the thinner $15 \mathrm{~nm}$ structure used for displays. The EML thickness has been adjusted to give comparable color in the two cases. The current efficacy (cd/A) is higher for the thick ETL, offset somewhat by the higher voltage. Overall, however, the $\mathrm{Im} / \mathrm{W}$ value was $10 \%$ higher for the thick ETL $(28 \mathrm{~lm} / \mathrm{W})$. Most importantly, the rectification ratios for the thick ETL devices are 200,000, more than 20x higher than the thinner ETL devices - indicating more robust devices, less prone to develop electrical shorts - thereby validating the original reason for choosing a thicker film structure.

One objection to the use of thick ETL devices for displays is the significant degree of color dispersion with viewing angle. As an example, Fig.18 shows this for a green device. However, this is not a concern for lighting structures used in this project due to the use of outcoupling enhancement films which effectively reduces this problem by randomizing the emission angle of light rays. This is clearly seen in Fig. 18.

One final comment relates to the selection of thick layers in these structures. As noted earlier, the absolute color of the three primaries used in the panels is not critical due to the ability to tune the overall color of the panel. Consequently we have more latitude to adjust the device cavity to maximize the luminance, with less concern for achieving a specific color-point. 

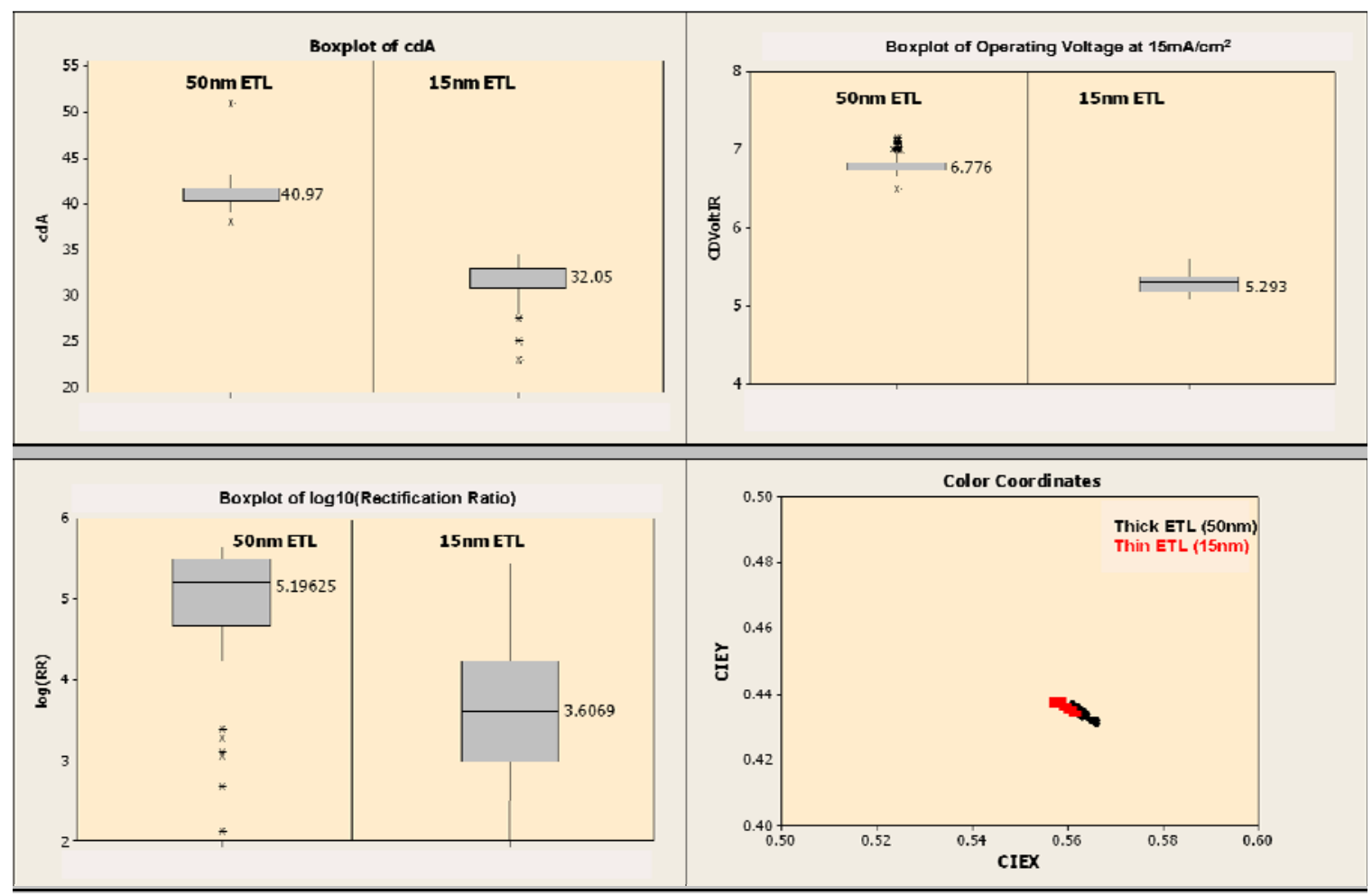

Fig.17. Comparison of 'Thick' (50nm) and 'Thin' (15nm) ETL structures for orange devices.

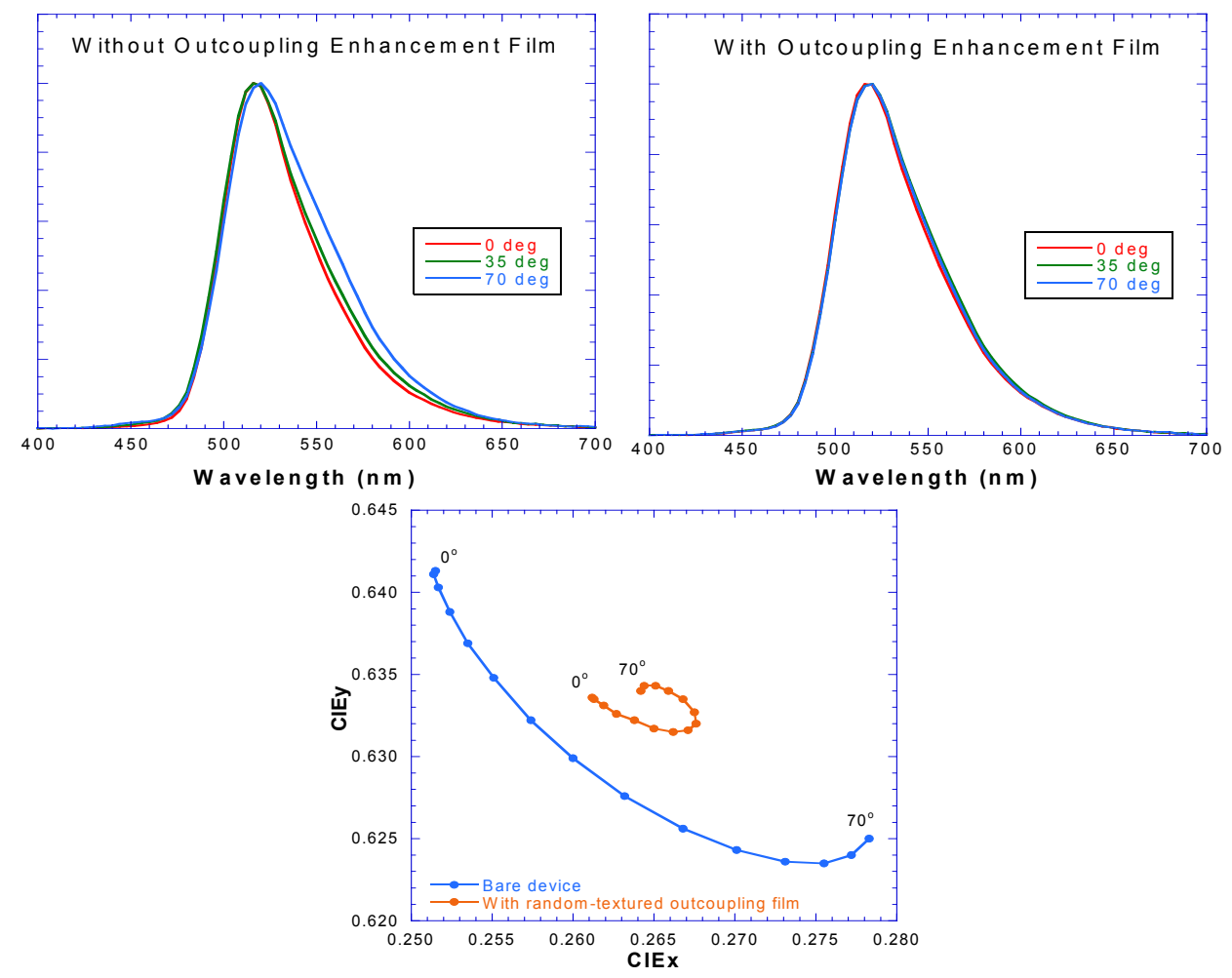

Fig.18. Demonstration, with green devices, that the use of a randomly textured outcoupling enhancement film effectively minimizes color-dispersion with viewing angle. 


\subsection{Use of an n-Doped Electron Transport Layer to Lower the Operating Voltage}

Minimization of the operating voltage is a key strategy in maximizing the $\mathrm{Im} / \mathrm{W}$ performance of a device, as well as for extending its operational lifetime. One effective way to achieve this is with molecular doping of the ETL layer. This is particularly important if the ETL layer is thick, as is the case for this architecture.

NDN-26 (NovaLED AG, Dresden), was selected as such a dopant and used successfully in these devices. It is codeposited, along with the ETL layer, in a vacuum deposition step at a doping level of $7.5 \%$ (by volume). This layer is deposited in a simple unpatterned layer with all colors having the same ETL layer thickness. The main drawback for this approach is the added cost of the NDN-26. However, the performance improvement is significant as can be seen in Fig.19. No additional benefit was found from increasing the doping levels beyond $7.5 \%$ (Fig.20).
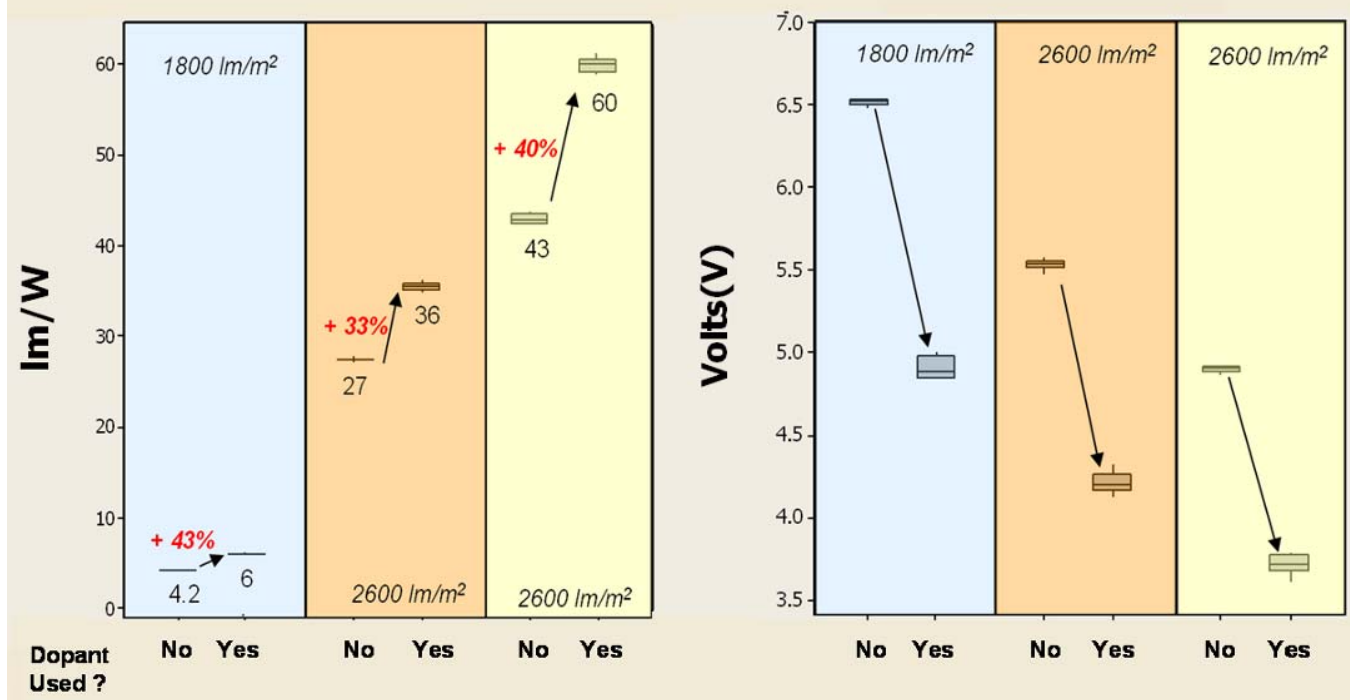

Fig.19. The doped ETL devices show a significant enhancement of the $1 \mathrm{~m} / \mathrm{W}$, primarily a result of the reduced operating voltage. These data were collected with printed $2 \mathrm{~cm}^{2}$ devices, but the benefits were demonstrated on mid-sized, $50 \mathrm{~cm}^{2}$ devices also.
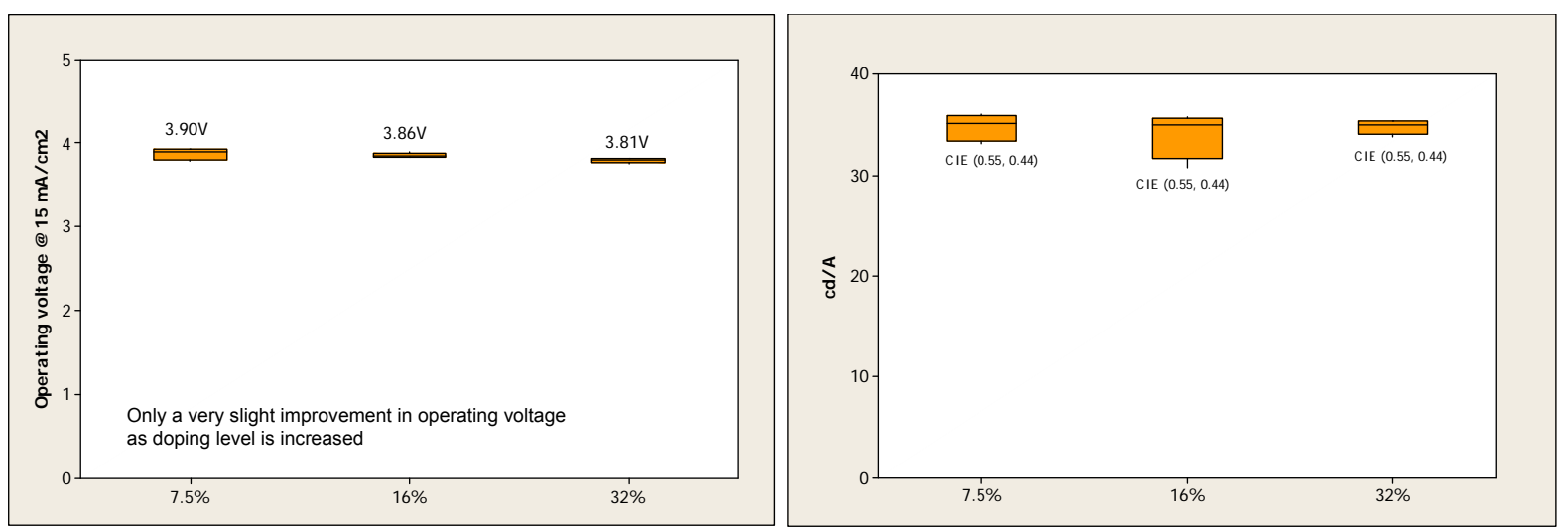

Fig.20. Little difference was seen in the color, operating voltage or current efficiency for devices having doping levels ranging from the recommended doping level ( 7.5\%) up to $4 x$ this level . 


\subsection{Outcoupling Enhancement Films}

Outcoupling enhancement films are routinely used to enhance the luminance of a lighting panel by extracting some of the light that is trapped in the glass substrate in the "substrate-guidedmode". A brief explanation of this phenomenon, and the benefit (and limitations) of using an enhancement film, is described in Fig.21.

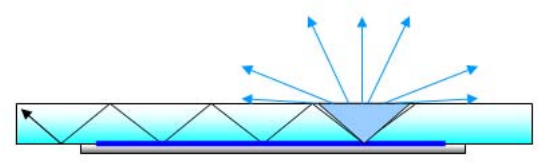

For a uniform planar glass substrate, rays which are emitted outside the escape-cone can never exit the device. They are subject to 'Total Internal Reflection'. Only $20-25 \%$ of rays have a sufficiently low angle to be able to exit. Most are trapped

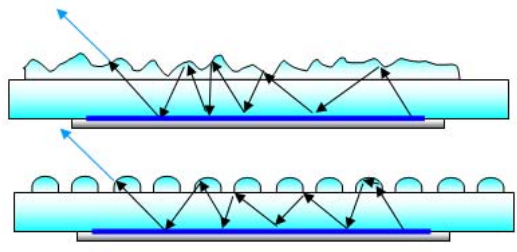
in the glass substrate and ultimately absorbed.

But if the glass surface is less regular, e.g. by using arrays of microprisms, or by roughening, light rays hit the surface at a range of angles. After multiple reflections the ray hits the surface at a sufficiently low angle (less than the escape cone angle) and can exit. This is a stochastic process.

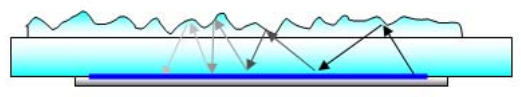

Unfortunately there is significant reabsorption of the photons by the OLED \& the cathode. Most photons get reabsorbed after a few reflections so we can't recycle all of them.

This limits the performance of the enhancement film.

Fig.21. A simplified explanation of the benefits (and limitations) of optical enhancement films.

In this project we assessed 15 different enhancement films (most of which are commercially available) to determine which performed best for our printed, pixellated panels. The main metric for assessing performance was luminance gain (integrated over all angles), but it was also important that color-dispersion with viewing angle was not significant. In addition, the degree of light directionality was assessed - although this was not used as a critical metric since lambertian emission might be desirable, or not, depending on the application.

The various enhancement films fell into four general categories: microlens arrays, regular prismatic arrays (typically used in LCD panels for light control), randomly textured films, and scattering films. These are discussed in more detail in Fig.22. Careful evaluation of these films, using statistical techniques, and with printed, yellow, $2 \mathrm{~cm}^{2}$ devices, gave the results shown in Fig.23. The most successful films (for this application - not necessarily a generality) were the randomly textured films. It was found that the higher the degree of texturing the greater the luminance enhancement. A gain of $35 \%$ was routinely achieved for the most textured films. Films provided by WaveFront Technology (CA, USA) and Kimoto Co. Ltd. (Japan) were statistically equivalent. Although this evaluation was performed with yellow devices, subsequent studies showed that a 35\% gain was similar for all colors (see Fig.24).

(It is important to note that values $>35 \%$ are achievable for sub-optimized device structures. However, the net performance of such devices is worse than those described here.)

Based on these data, textured films were selected for use in this project. WaveFront technology films, and Kimoto films, being equivalent, were both used. 


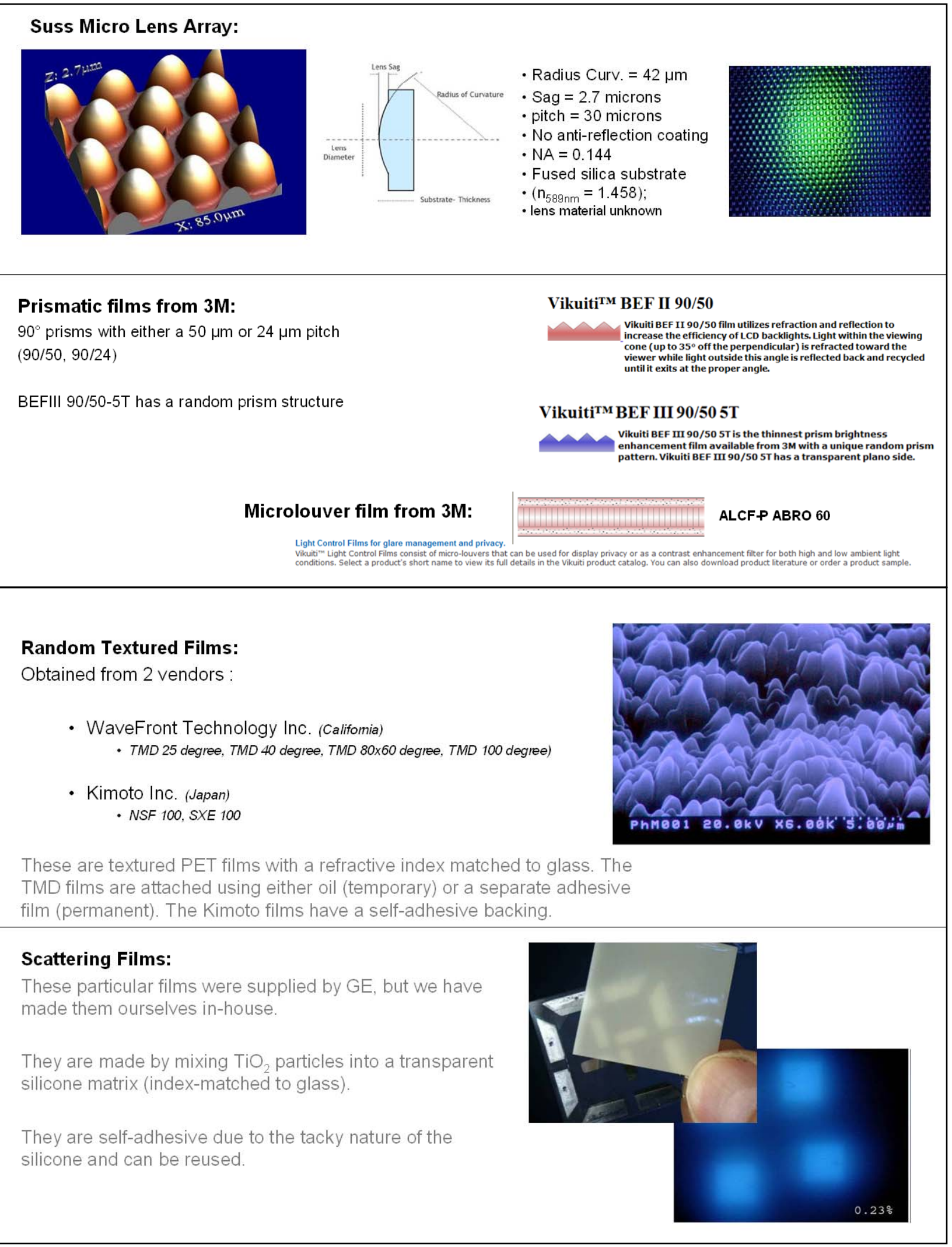

Fig.22. The various types of enhancement films evaluated in this project. 


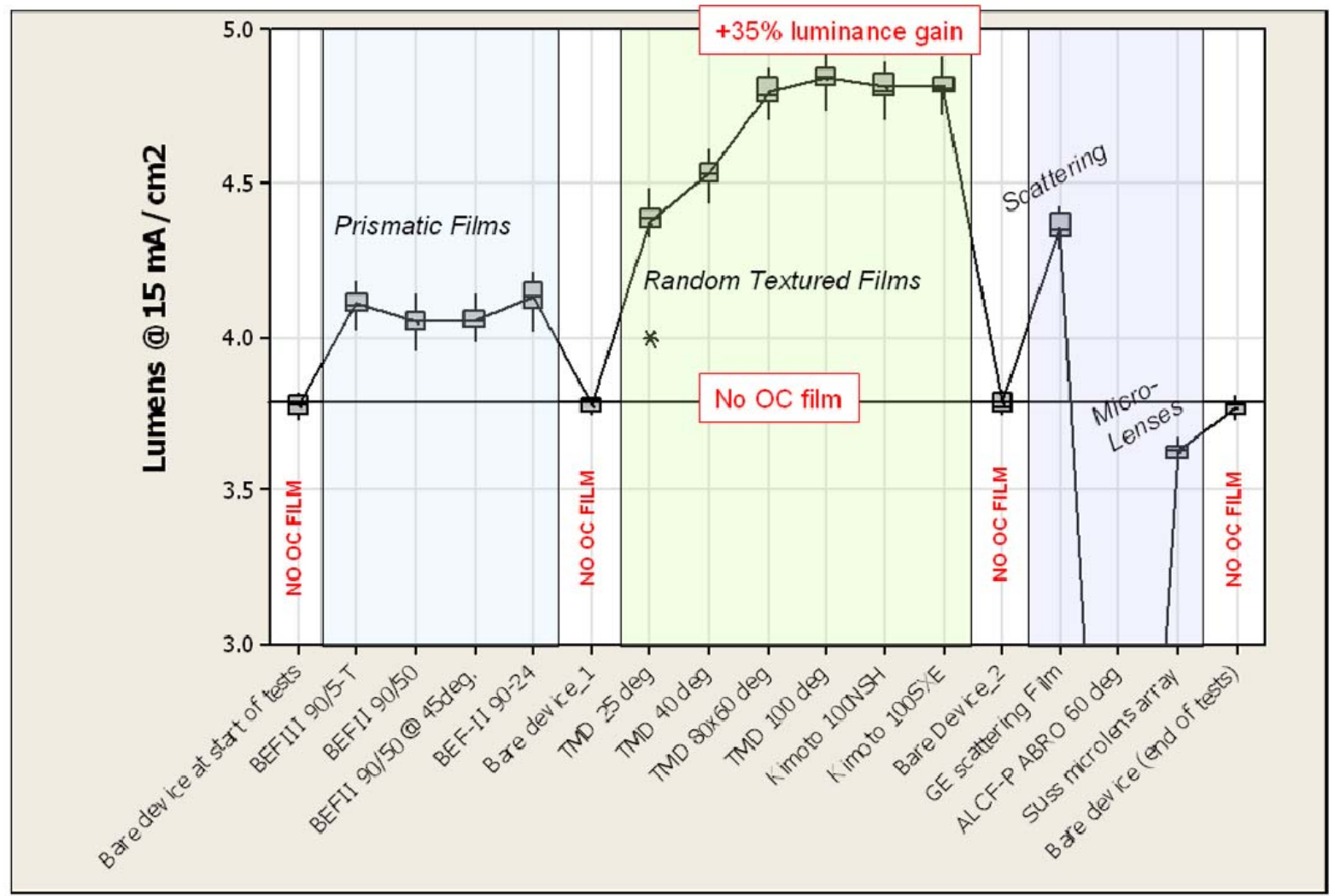

Fig.23. Luminance for printed yellow $2 \mathrm{~cm}^{2}$ devices, with and without enhancement films ("OC" refers to "outcoupling" film). The same set of 16 devices was used to evaluate each enhancement film - thereby ensuring relative accuracy.

\begin{tabular}{|c|c|c|c|c|c|c|c|c|}
\hline \multicolumn{2}{|c|}{$\begin{array}{l}\text { All devices are Printed } \\
\text { Cham ILP with WFT } 100^{\circ} \mathrm{TMD} \text { film }\end{array}$} & \multicolumn{5}{|c|}{$\begin{array}{l}\text { Measured in Integrating Sphere } \\
\text { (only considering front emission) }\end{array}$} & \multicolumn{2}{|c|}{$\begin{array}{l}\text { Meas. with Photometer } \\
\text { at } 1000 \mathrm{~cd} / \mathrm{m}^{2}\end{array}$} \\
\hline & CIE & $\begin{array}{l}\text { Meas. } \\
\text { condition }\end{array}$ & $\begin{array}{l}\text { Lm/W } \\
\text { with OE }\end{array}$ & $\begin{array}{c}\% \text { gain } \\
\text { with OE }\end{array}$ & $\begin{array}{l}\text { EQE (\%) } \\
\text { with OE }\end{array}$ & $\begin{array}{c}\% \text { gain } \\
\text { with OE }\end{array}$ & $\begin{array}{c}\text { Cd/A } \\
\text { with OE }\end{array}$ & $\begin{array}{l}\% \text { gain } \\
\text { with OE }\end{array}$ \\
\hline Deep red & $(0.645,0.354)$ & $2100 \mathrm{Im} / \mathrm{m}^{2}$ & 10.3 & +37 & 16.8 & +26 & 22.1 & +46 \\
\hline Orange & $(0.560,0.438)$ & $"$ & 30.0 & +35 & 21.6 & +28 & 55 & +37 \\
\hline Yellow & $(0.471,0.522)$ & $2600 \mathrm{Im} / \mathrm{m}^{2}$ & 45 & +33 & 21.2 & +26 & 78 & +28 \\
\hline Green & $(0.266,0.629)$ & $"$ & 11.5 & +34 & 5.8 & +27 & 23 & +30 \\
\hline Pale Blue & $(0.137,0.171)$ & $1800 \mathrm{Im} / \mathrm{m}^{2}$ & 4.3 & +43 & 6.7 & +40 & 10 & +54 \\
\hline Deep Blue & $(0.140,0.140)$ & $"$ & 3.0 & +33 & 4.3 & +21 & 6.2 & +17 \\
\hline
\end{tabular}

Fig.24. Performance data for printed $2 \mathrm{~cm}^{2}$ area devices, with a Wavefront Technology $100^{\circ} \mathrm{TMD}$ randomly textured outcoupling film. The enhancement is effective for all colors, typically giving a $35-40 \%$ gain in Lm/W. ("OE" means "outcoupling enhancement".) 


\section{Device Performance}

\subsection{Color Selection:}

As discussed earlier, materials for this project were selected based on high efficacy, rather than for saturated colors. The specific colors of the materials are not critically important (since the device is color tunable), as long as the color-gamut (indicated by the dotted triangle in Fig.25) encompasses the desired colors - in this case points along the black-body locus between 2600K and 6500K (illuminant-A and D65).

After evaluation of a range of emitters chosen from DuPont Displays' library of OLED materials, a final selection of a high efficiency orange emitter (CIE cords : $(0.56,0.44)$ ), a high efficiency yellow $(0.47,0.52)$, and a fluorescent blue $(0.14,0.19)$ was made. These materials were previously known (or were measured as part of this project) to have T70 lifetimes in excess of the $5,000 \mathrm{hr}$ target for this project. Actual device lifetime data is discussed in detail in section 8.6.

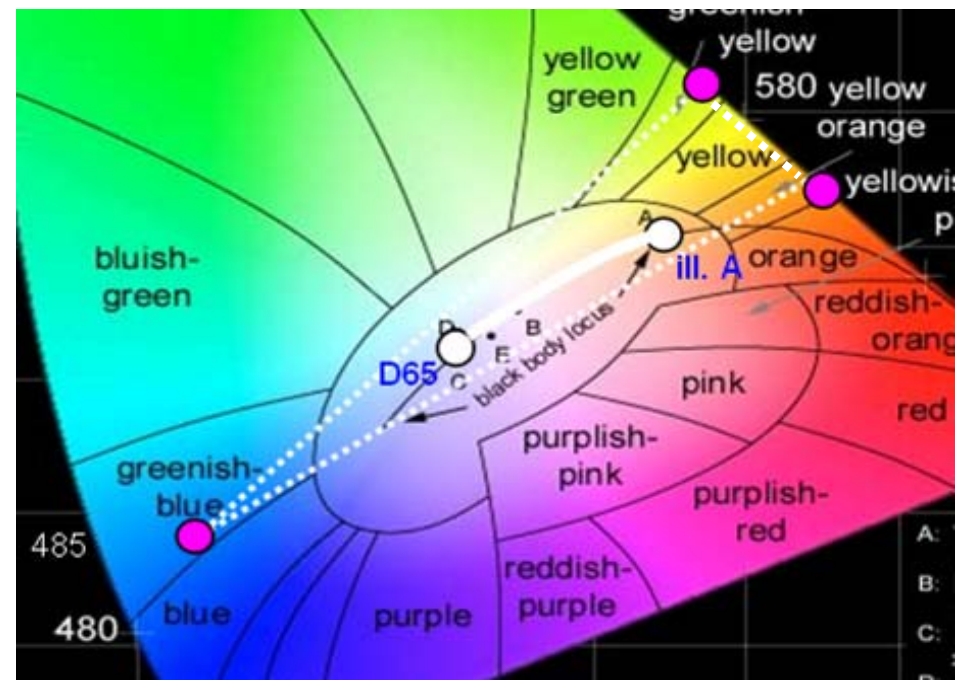

Fig.25. The color primaries and color-gamut chosen for this project, along with the desired range for white-point tunability.

\begin{tabular}{|c|c|c|c|c|}
\hline & CIE & Lm/,000 cd/m² & $\begin{array}{c}\text { EQE } 6 \\
1,000 \mathrm{~cd} / \mathrm{m}^{2}\end{array}$ & $\begin{array}{l}\text { T70 Lilietime in a } \\
1,000 \mathrm{~cd} / \mathrm{m}^{2} \text { white } \\
\text { Pans (lllum.-A) }\end{array}$ \\
\hline Orange & $(0.56,0.44)$ & 33 & $20 \%$ & $80 \mathrm{khrs}$ \\
\hline Yellow & $(0.47,0.52)$ & 55 & $22 \%$ & $>56 \mathrm{khrs}$ \\
\hline Pale Blue & $(0.14,0.19)$ & 5 & $6 \%$ & $>10 \mathrm{khrs}$ \\
\hline
\end{tabular}

Fig.26. Color and efficiency data are from $50 \mathrm{~cm}^{2}$ emissive area printed devices with WaveFront Technology TMD100 outcoupling enhancement film. Data are measured in an integrating sphere. Lifetime data is from test coupons under accelerated test conditions (see section 8.6) 
The benefit of using high efficiency emitters is clear from the table, with yellow and orange materials having external quantum efficiency values (EQE) $\geq 20 \%$ - compared with only $6 \%$ for the fluorescent blue emitter. This $3-4 x$ difference is expected, and is exacerbated by the poor eye response for blue light, so that the $\mathrm{Im} / \mathrm{W}$ value for blue is more than $10 x$ lower than for yellow. This combination of emitters, in a $50 \mathrm{~cm}^{2}$ panel, gives performance in the range of 33-35 $\mathrm{Im} / \mathrm{W}$ at $1,000 \mathrm{c} / \mathrm{m}^{2}$, for white at illuminant-A (see Fig.27).

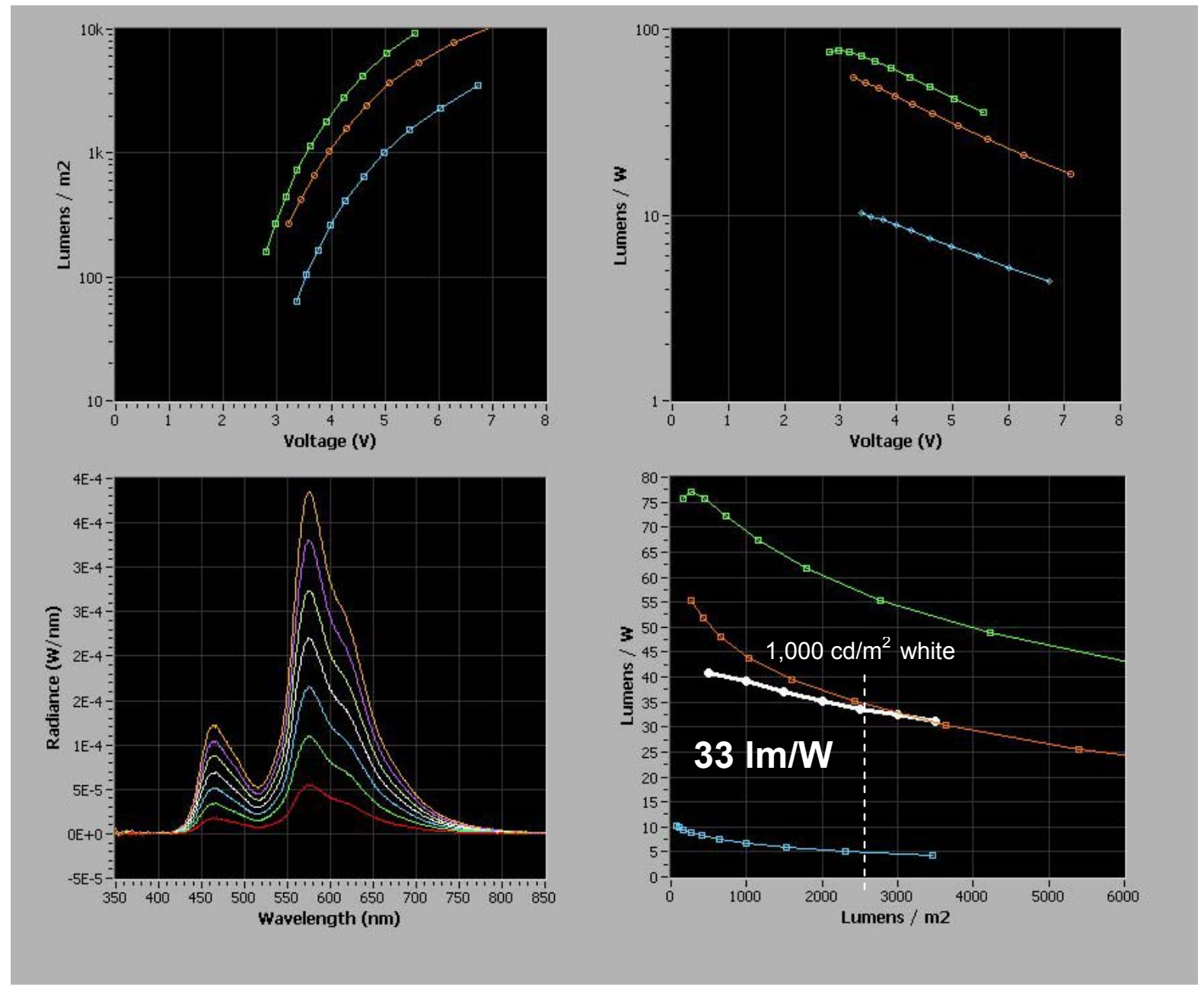

Fig.27. Typical performance for a $50 \mathrm{~cm}^{2}$ white-panel as measured in an integrating sphere. The performance of the three individual color primaries is shown, along with white tuned to illuminant-A, for which the combined efficacy $\left(\right.$ at $\left.1,000 \mathrm{~cd} / \mathrm{m}^{2}\right)$ is $33-35 \mathrm{Im} / \mathrm{W}$. 


\subsection{Efficacy and Lm/W Contours:}

Since we have a color-tunable device, there is no unique efficacy value - it depends upon the chosen color. Fig. 28 shows the $1 \mathrm{~m} / \mathrm{W}$ contours for the devices described above. As expected, the efficacy decreases for higher color-temperatures as a greater proportion of blue is required for these whites.
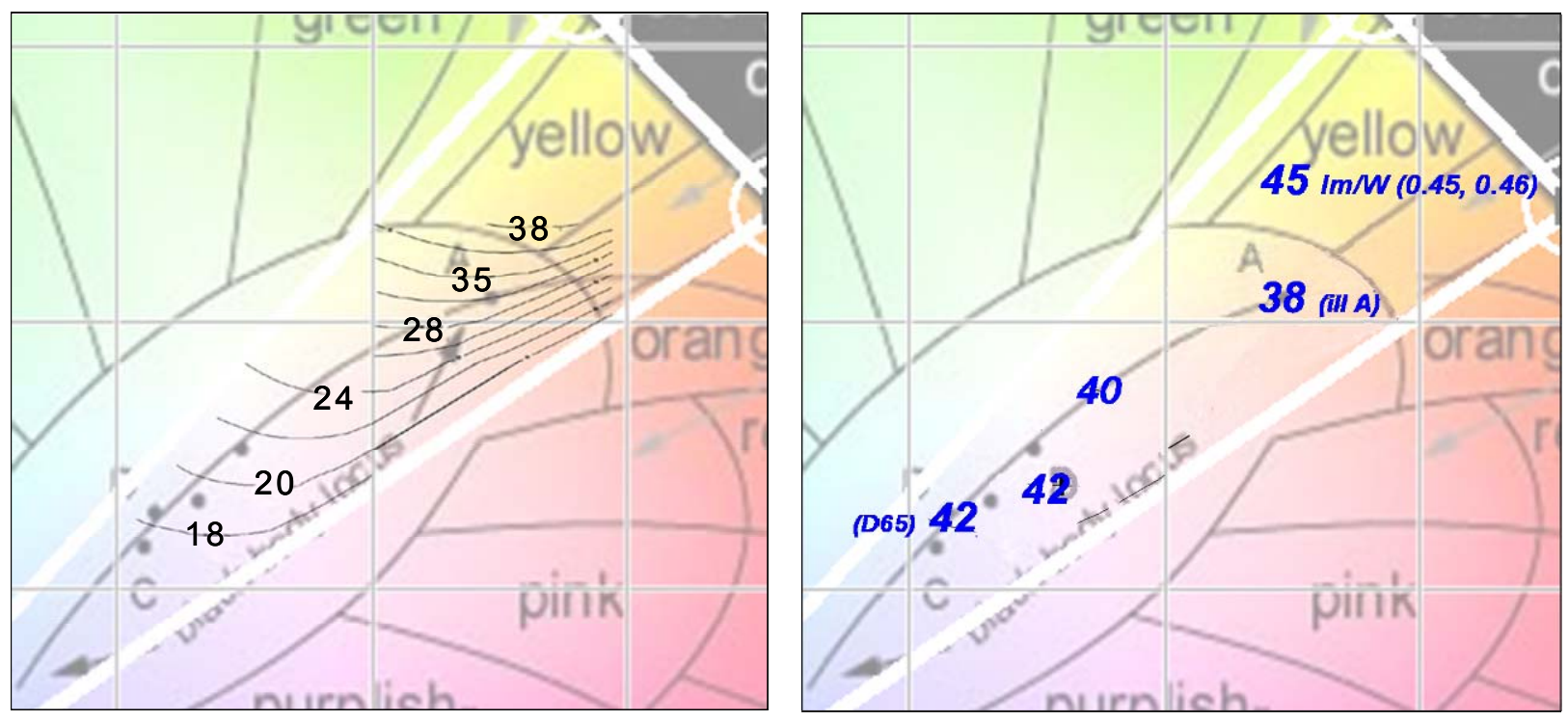

(Left) Fig.28. $L m / W$ contours for the color tunable devices

(Right) Fig.29. Modeled Lm/W values for a hypothetical high efficiency blue having a $20 \%$ EQE, used in combination with the orange and yellow materials described in the text.

\subsection{Discussion of the Limited Blue Performance}

This performance level is less than the target value of $40 \mathrm{~lm} / \mathrm{W}$, and is primarily a result of the underperforming blue material which lowers the overall performance. This becomes apparent when we model the performance we might expect to achieve if the fluorescent blue were exchanged with a high efficiency blue having an EQE comparable to the other materials (i.e. $20 \%$ EQE). As can be seen in Fig.29, we would expect to reach $~ 40 \mathrm{Im} / \mathrm{W}$ performance across the whole range of the target colors. As part of this project several high efficiency blue emitters were evaluated, in an attempt to rectify this poor $\mathrm{Im} / \mathrm{W}$ performance for blue. This work is discussed in detail in section 8.7. 


\subsection{Color Rendering Index:}

Fig.30 shows the variation in the Color Rendering Index (CRI) at various white-points. For the 'cooler' whites the CRI is in the range of $78-83$, however, this is reduced at both higher and lower color-temperatures, decreasing to only 65 near to illuminant-A. The reason is clear if the emission spectrum of the panel is reviewed (Fig.31). The similarity of the wavelength maxima for the orange and yellow emitter means that there is a lack of green in the emission spectrum thereby lowering the CRI for 'warm' whites. For the 'cooler' whites, the increased contribution of blue balances the spectrum to give higher CRI values.

This problem can be addressed by using a 'yellow-green' emitter instead of the regular yellow. However, it is not a trivial problem since a greener primary requires the orange primary to be operated at a higher luminance (to achieve the same white-point), which moves its operating point to a lower $\mathrm{Im} / \mathrm{W}$ value. Care is needed to strike a balance between improved CRI and decreased $\mathrm{Im} / \mathrm{W}$. Another possibility is to use a yellow emitter with an inherently broader spectrum. Preliminary work was started to explore these possibilities, but this work remains to be completed.

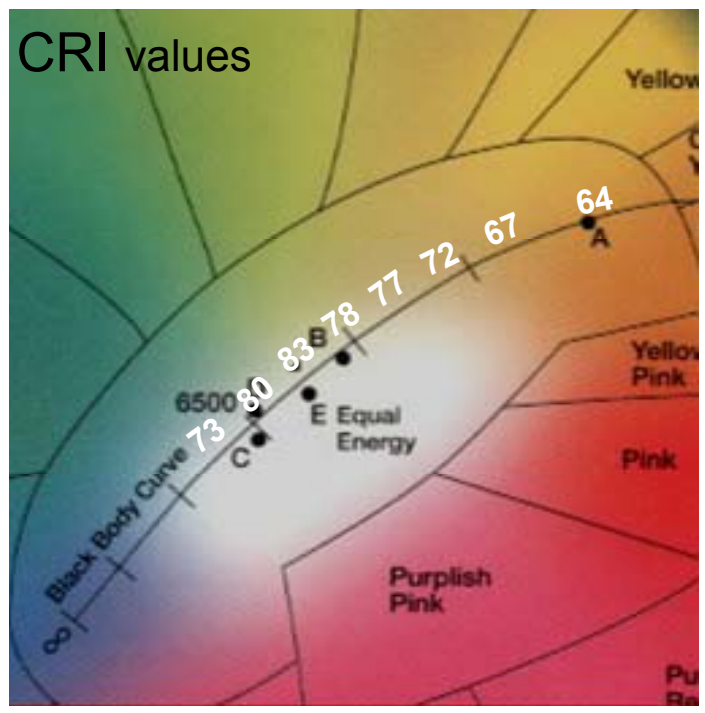

Fig.30. CRI of a panel at various white-points


Fig.31. (Left) Panel spectrum at D65, showing a CRI of 79; (Right) Panel spectrum at illuminant-A, showing a CRI of 65. 


\subsection{Color Dispersion:}

Fig.32 shows the angular dispersion of the optical properties of a printed $50 \mathrm{~cm}^{2}$ panel, with an outcoupling enhancement film (WaveFront Technology TMD 100). There is a slight 'blue-shift' at higher angles, although the effect is minor and barely noticeable to the eye. Overall this panel shows close to 'Lambertian' behavior, which is desirable for an indoor general lighting application.
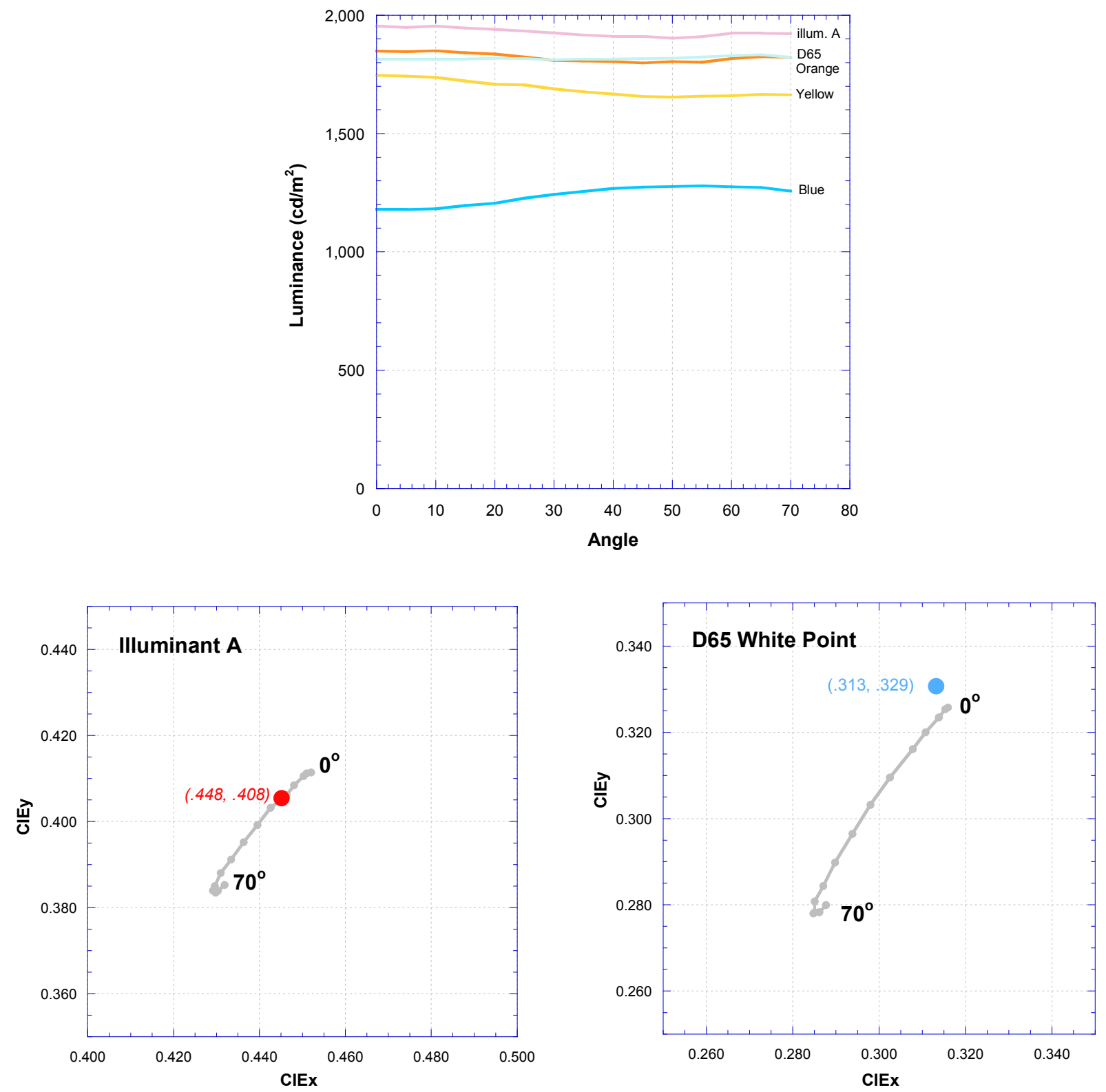

Fig.32. The angular dispersion of luminance and color coordinates for the primary colors of the OLED panel, and for two of the target white-points. The angle is with respect to the normal of the panel. 


\subsection{Device Lifetime}

\section{Blue Devices}

Raw lifetime data for printed blue devices is shown in Fig.33. This data was collected by operating hermetically sealed devices at a constant current in a temperature controlled chamber. For compatibility with the displays program at $D D I$, these values were set to $6.75 \mathrm{~mA} / \mathrm{cm}^{2}$ and $32^{\circ} \mathrm{C}$. The raw T70 lifetime is $3000-4000 \mathrm{hrs}$.
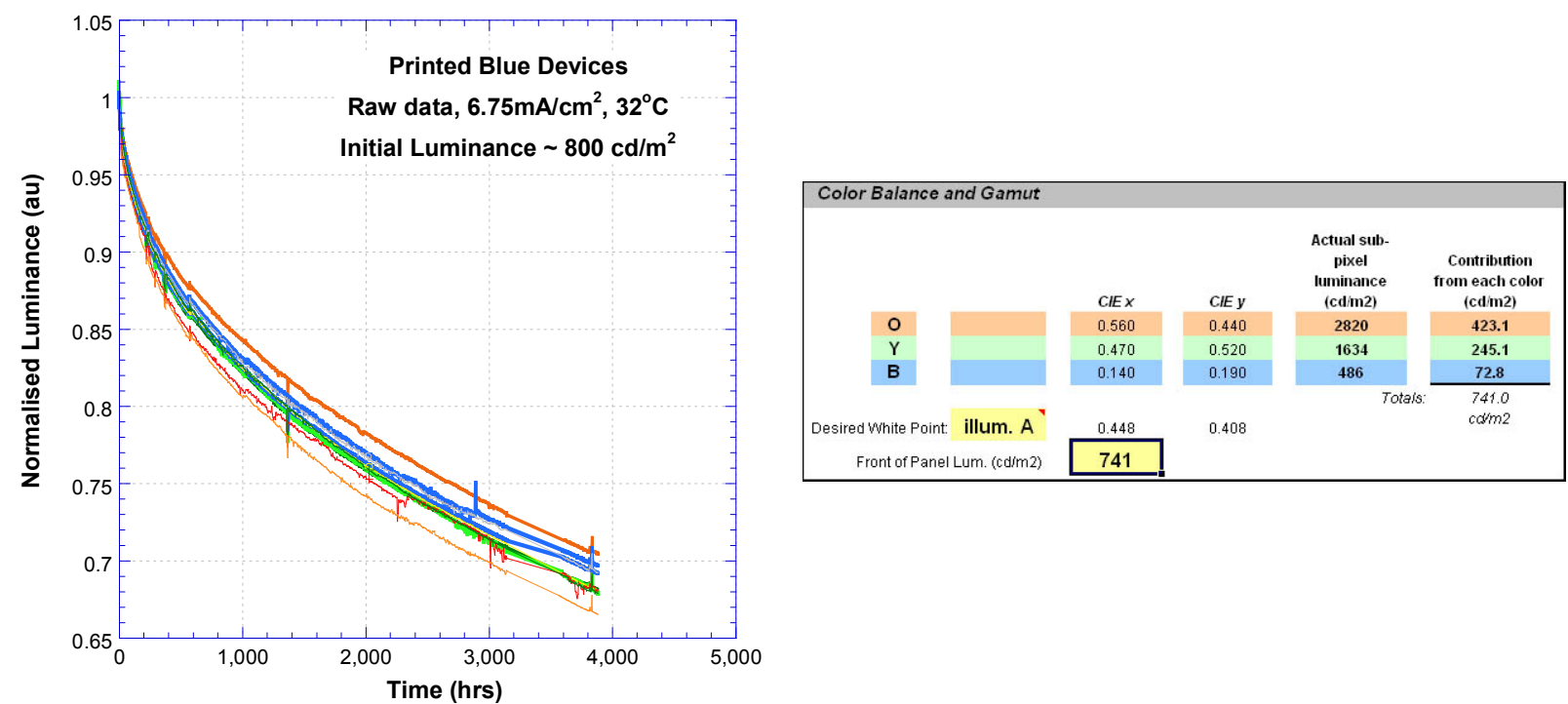

Fig.33. Raw lifetime data for printed blue devices, and the necessary scaling factors for a white panel operating under 'standard' conditions.

It is desirable to standardize these $\mathrm{T} 70$ data to $25^{\circ} \mathrm{C}$ and to a luminance level equivalent to that of a 'white' lighting panel operating at $1,000 \mathrm{~cd} / \mathrm{m}^{2}$ (illuminant-A.).

The temperature conversion factor has been accurately determined for this material and reducing the temperature from 32 to $25^{\circ} \mathrm{C}$ increases T70 by $1.49 x$ (i.e. to $4500-6000 \mathrm{hrs}$.).

We can calculate the operating luminance for the blue pixels in a lighting panel using the model discussed in section 5. We will assume that the panel is operating at 'illuminant-A' and at 1000 $\mathrm{cd} / \mathrm{m}^{2}$, with an outcoupling enhancement film having a $35 \%$ gain (see section 7.4 ). This means that the underlying panel has a white-luminance of $741 \mathrm{~cd} / \mathrm{m}^{2}$, for which the spatially averaged blue contribution is $72.8 \mathrm{~cd} / \mathrm{m}^{2}$. Considering that the blue sub-pixels only occupy $1 / 3$ of the active area, and that the fill factor for each sub-pixel is only $45 \%$, the actual 'in-pixel' blue luminance level is $486 \mathrm{~cd} / \mathrm{m}^{2}$ (see Fig.33).

The raw data presented above has the device operating (initially) at $800 \mathrm{~cd} / \mathrm{m}^{2}$, i.e. too high by a factor $(800 / 486)=1.65 x$. For simplicity we will assume that the device efficacy is the same at both the luminance levels (a conservative assumption), hence the currents in the 2 cases differ by $1.65 x$ also. The current correction factor has also been accurately determined for this material and reducing the current by $1.65 x$ increases T70 by $(1.65)^{n}$, where $n$ is $\sim 1.7$. Hence we find that the corrected lifetime is $2.3 x$ longer.

Combining these two corrections we estimate the 'standardized' T70 lifetime to be between 10,500 and 13,800 hrs. 


\section{Yellow Devices}

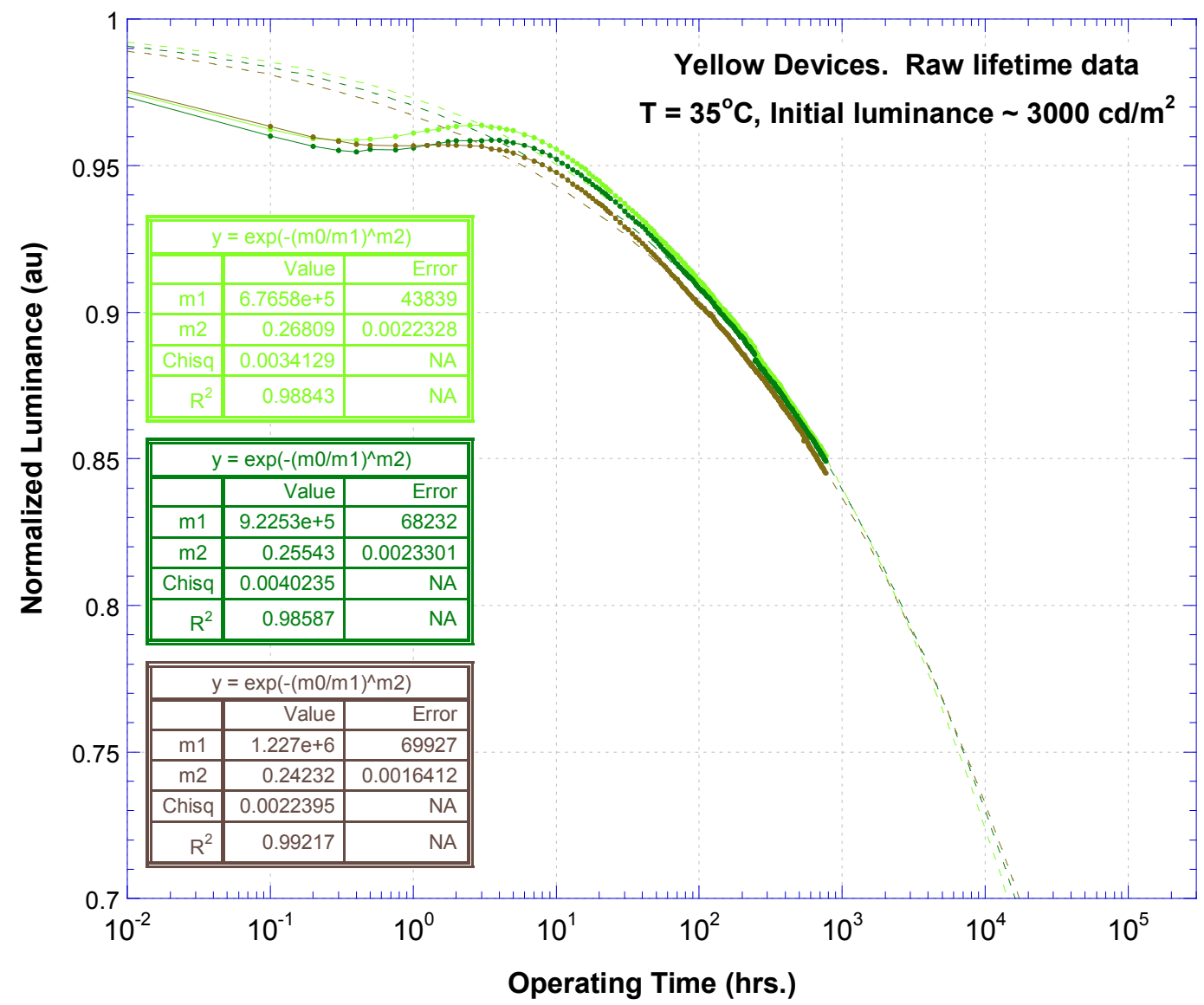

Fig.34. Raw lifetime data for yellow devices. Extrapolation uses a 'stretched exponential' type fit.

Fig.34 shows the raw lifetime data for yellow devices tested at $35^{\circ} \mathrm{C}$, with an initial luminance of $\sim 3000 \mathrm{~cd} / \mathrm{m}^{2}$. Yellow has a considerably longer lifetime than the blue, necessitating an extrapolation to estimate the T70 lifetime. It is found (empirically) that OLED device luminance frequently follows a 'stretched exponential' type dependence, and using such a fit we estimate $\mathrm{T} 70$ to be $\sim 10 \mathrm{k}-15 \mathrm{khrs}$. (The parameters of the fit are shown in Fig.34. $\mathrm{R}^{2}$ values are 0.99 . The less than perfect fit during the first hour is, at least partly, due to temperature equilibration of the test system.)

As with blue, it is desirable to correct this lifetime to 'standard conditions' of $25^{\circ} \mathrm{C}$ and $1,000 \mathrm{~cd} / \mathrm{m}^{2}$ 'white' (illuminant-A.). Although known with less certainty than for blue devices, the temperature correction factor from $35^{\circ} \mathrm{C}$ to $25^{\circ} \mathrm{C}$ is close to $2 x$ (consistent with activated behavior found for most OLED materials). From Fig.33 we see that the 'in-pixel' luminance level should be $1634 \mathrm{~cd} / \mathrm{m}^{2}$. Hence, using the same methodology as for blue, we calculate a luminance correction factor of $(3000 / 1634)^{1.7}=2.8 x$. Combining these corrections we project T70 to be in the range of $56,000-84,000 \mathrm{hrs}$. These numbers should be treated with some caution, due to the extended extrapolation and possible errors incurred by the corrections, however, it is very probable that T70 exceeds the target lifetime of 5,000hrs. 


\section{Orange Devices}

Fig. 35 shows the raw lifetime data for orange devices tested at $35^{\circ} \mathrm{C}$, with an initial luminance of $\sim 1300 \mathrm{~cd} / \mathrm{m}^{2}$. As with the yellow devices we use a 'stretched exponential' extrapolation and thereby estimate the raw T70 to be $\sim 150 \mathrm{khrs}$. (The parameters of the fit are shown in Fig.35. $\mathrm{R}^{2}$ values are 0.99.)

Again we correct this lifetime to 'standard conditions' of $25^{\circ} \mathrm{C}$ and $1,000 \mathrm{~cd} / \mathrm{m}^{2}$ white at (illuminant-A). Again, the temperature correction factor from $35^{\circ} \mathrm{C}$ to $25^{\circ} \mathrm{C}$ is close to $2 \mathrm{x}$. From Fig. 33 we see that the 'in-pixel' luminance level should be $2820 \mathrm{~cd} / \mathrm{m}^{2}$. Hence, using the same methodology as before, we calculate a luminance correction factor of $(1300 / 2820)^{1.7}=0.27 x$. Combining these corrections we project T70 to be $\sim \mathbf{8 0 , 0 0 0}$ hrs. Again, this number should be treated with some caution, but it is very probable that T70 exceeds the target lifetime of $5,000 \mathrm{hrs}$.



Fig.35. Raw lifetime data for orange devices. Extrapolation uses a 'stretched exponential' type fit.

Overall, all three materials have T70 values that are estimated to considerably exceed the project target of $5000 \mathrm{hrs}$. As expected, the blue material has the shortest lifetime, and since it differs so considerably from the orange and yellow it might be anticipated that the panel will 'color-shift' as it ages. However, one significant advantage of our tunable-color architecture is that it is trivial to rebalance the colors and reestablish the 'white-point' should it prove to be necessary. This could be achieved manually using a 'trim-pot' on the blue channel. Alternatively, in a more demanding application, using a controller with color sensor feedback, this could be automated. 


\subsection{Evaluation of High Efficiency Blue Materials}

High efficiency blue emitters from DuPont Displays' library of emitters were carefully considered for this project, but it was already known from previous testing that their lifetimes were generally too short compared to the $5,000 \mathrm{hr}$. target for this project. The development of new materials was not a part of this project. However, development of high efficiency blues is an important part of $D D I$ 's displays program, and new materials were evaluated for suitability for lighting as they became available. In addition, we purchased 2 materials from American Dye Source (Quebec, Canada) for evaluation and comparison. Both of these materials were extensively repurified by train-sublimation prior to use.

- ADS065BE bis(2-(4,6-difluorophenyl)pyridinato-N, $\left.C^{2}\right)$ picolinate iridium (III), more commonly known as firpic

- ADS070BE tris(2-(2,4-difluorophenyl)pyridine)iridium(III)

It should be noted that switching from a fluorescent to a high efficiency emitter requires a different choice of materials for other layers in the device too - the HTL layer and hosts of the EML layer - materials that must have triplet levels matched to the high efficiency dopant. Such materials were available for use from DDI's materials library, and had been previously tested.

The results of extensive testing of these ADS materials showed that firpic was much more efficient than ADS 070, able to reach an EQE of $(\sim 9 \%)$ and $\sim 12 \mathrm{~lm} / \mathrm{W}$ (with outcoupling enhancement), so most of the subsequent work focused on this material.
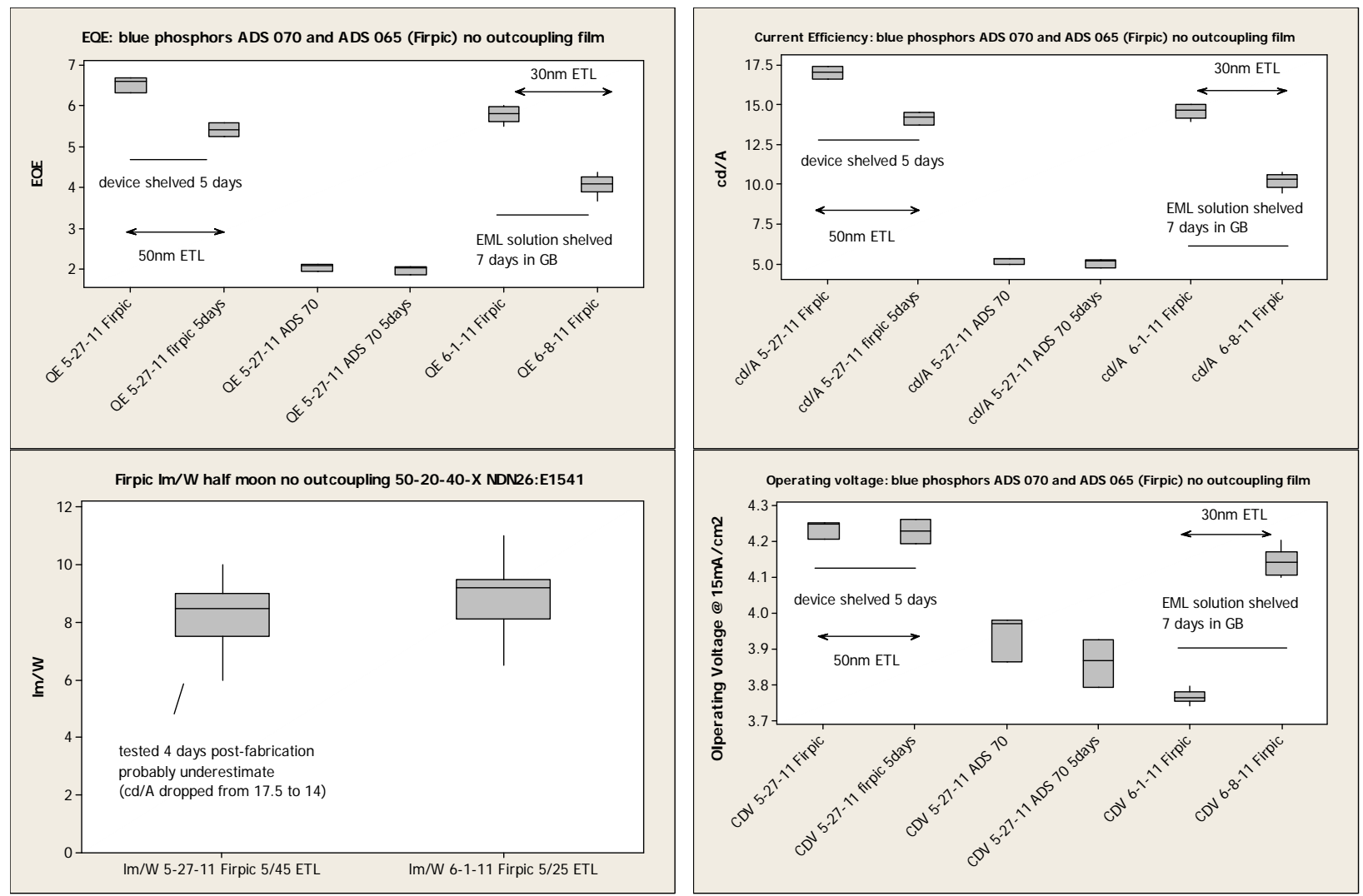

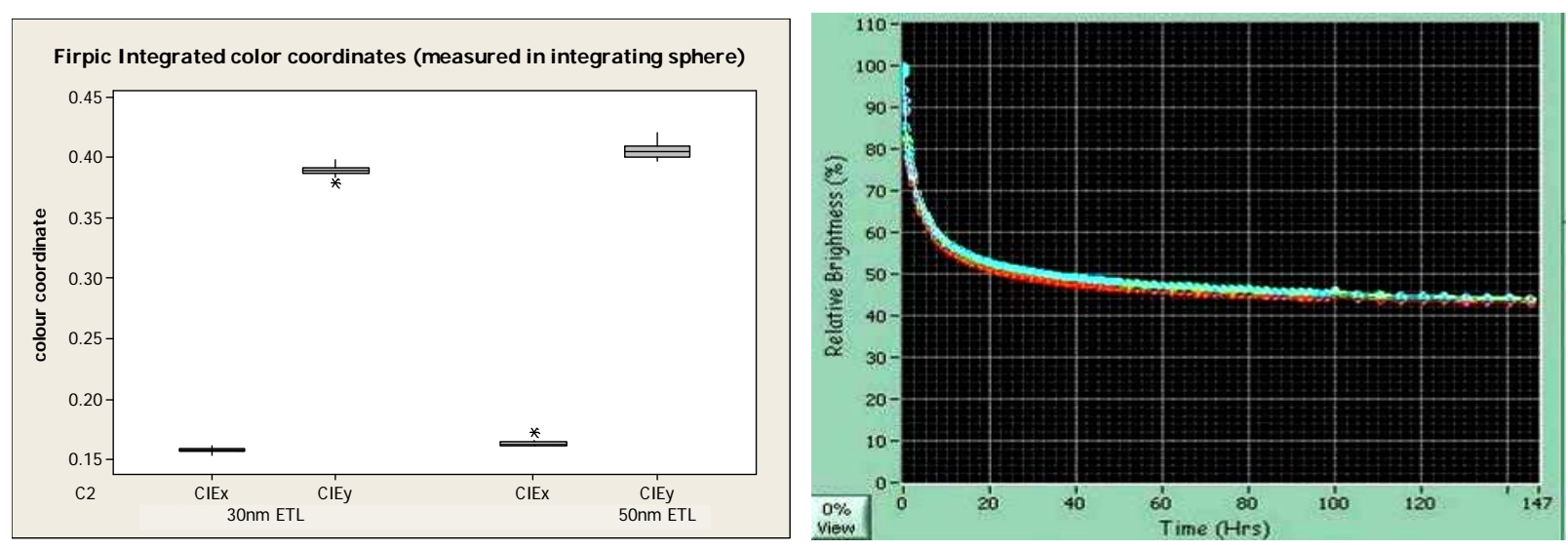

Fig.36. Typical performance data for devices fabricated using the high efficiency blue 'firpic' from American Dye Source. In general the solutions and devices had poor stability.

Unfortunately, stability of this material was found to be a problem, both for devices (under storage and stress conditions) and of the solutions used to make the devices: (i) stress lifetime was very short - at $1000 \mathrm{~cd} / \mathrm{m}^{2}, \mathrm{~T} 50$ was reached in little over 20 hours (ii) within devices, the EML was unstable - the efficiency dropped by more than $20 \%$ after 5 days of shelf-storage (the operating voltage remained stable during this period, however, which suggests that the other materials in the structure were stable) (iii) the EML solution was unstable - a $50 \%$ drop in efficiency was observed for EML stored in an inert $N_{2}$ glovebox for 1 week.

Another problem with firpic relates to its color. With a CIEy value close to 0.40 , the black-body locus mostly falls outside of our color gamut, so we cannot generate illuminant-A or D65. Ideally we need a high efficiency blue with a CIEy value in the range of $0.11-0.25$. (See Fig.37). Unfortunately most currently available high efficiency blues are too green for us to use including Universal Display Corporations' high efficiency blue emitter $(0.18,0.42)$.



Fig.37. Commercially available high efficiency blue emitters, including firpic, are generally too green for use in this project - the resulting color gamut would exclude most of the black-body locus of 'whites' particularly those range of color temperatures of most interest here. It can be seen that the highest practical CIEy value, which would allow us to be able to tune to $D 65$, is 0.25 . Ideally the material would be bluer still, with a value in the range of 0.11 -0.25 . The DuPont fluorescent blue used for this project has an ideal color: CIEy $\sim 0.19$. 


\subsection{Optimizing ITO thickness}

DDI's standard display fabrication platform uses $180 \mathrm{~nm}$ ITO on borosilicate glass substrates. As mentioned before, in order to be consistent with pilot-line fabrication procedures, it was decided to adopt as much of the same panel architecture as possible, including the substrate / ITO components.

However, 180nm ITO is not ideal for lighting panels. Based on the architecture described previously, our models suggested that a thinner ITO, would work better, with $110 \mathrm{~nm}$ being optimal. This was supported by the performance of test coupons on a range of ITO thicknesses. This data is shown in Fig.38. The test coupons suggested that a gain of $\sim 20 \%$ could be achieved by switching to the thinner ITO. This held out the promise of boosting device performance from $35 \mathrm{Lm} / \mathrm{W}$ to $42 \mathrm{Lm} / \mathrm{W}$.

In practice we encountered problems when we tried to implement $110 \mathrm{~nm}$ ITO for the $50 \mathrm{~cm}^{2}$ panels. 6" plates were custom coated with 110nm ITO, by an outside vendor. However, the ITO etched unevenly giving poor quality devices. No obvious defects were present on the substrates prior to etching, and the suspicion was that the problem was caused by local variations in ITO chemistry leading to different etch rates. Unfortunately due to the turnaround time for this process we were unable to repeat the work before the end of the project. None-the-less, it is anticipated, based on the small coupons, that this is a feasible approach, and could be made to work on the larger panels, with further effort.
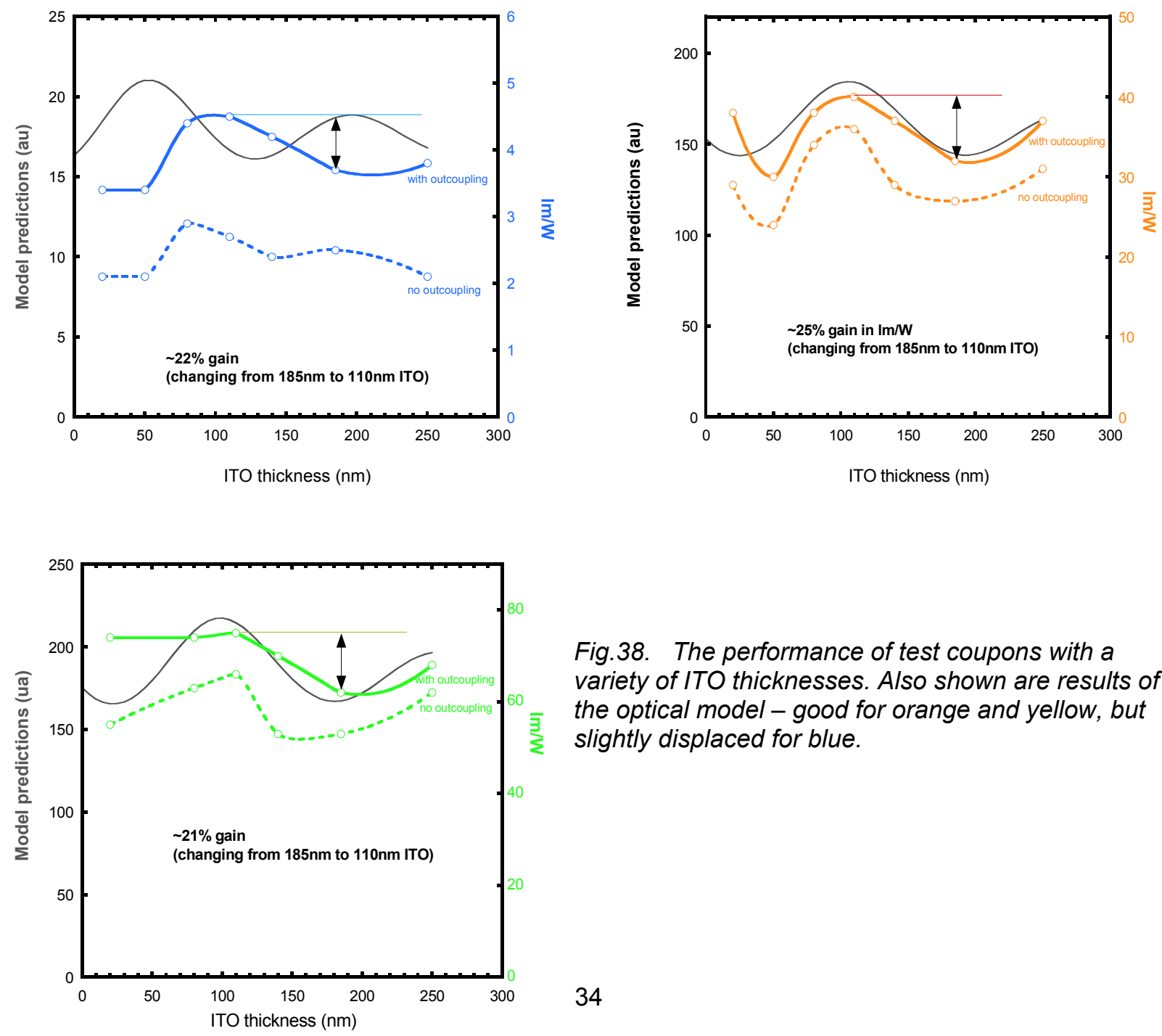

Fig.38. The performance of test coupons with a variety of ITO thicknesses. Also shown are results of the optical model - good for orange and yellow, but slightly displaced for blue. 


\section{Device Engineering}

\subsection{Mechanical Layout of the Lighting Panel}

The mid-sized panel design has an emissive area of $50 \mathrm{~cm}^{2}$ and is processed as two panels per 6 " square glass substrate. These substrates have been pre-patterned to include ITO/metal bus-lines (to carry the current to the pixels), other metallic lines to gang all the corresponding columns together, the ITO active area, and the ITO 'limit resistors' (described in section 7.1). A schematic is shown in Fig.39. At the plate level (6" $\times 6$ "), there are additional test structures for allowing process checks. After encapsulation of the panels, these $6 \times 6$ plates are scribed and cut into two individual panels.

Within the active area of the device, the pixel dimensions are 462x462 microns (giving a 55ppi resolution), with each sub-pixel being 1/3 the width, i.e. 154 microns wide. There are 103 rows and 579 columns of sub-pixels, giving a total of $\sim 60,000$ sub-pixels per panel. The $55 \mathrm{ppi}$ resolution is not specific to this lighting panel design, and other resolutions would work equally well, particularly since the use of the outcoupling enhancement layer 'blurs' any discrimination of the pixels. This design was chosen as a convenient platform to use based on previous display panel prototypes, for which a successful process had been developed. One minor drawback to this design is that the active area only occupies $45 \%$ of the panel (as mentioned earlier there is dead space which would be occupied by transistor structures in a display).

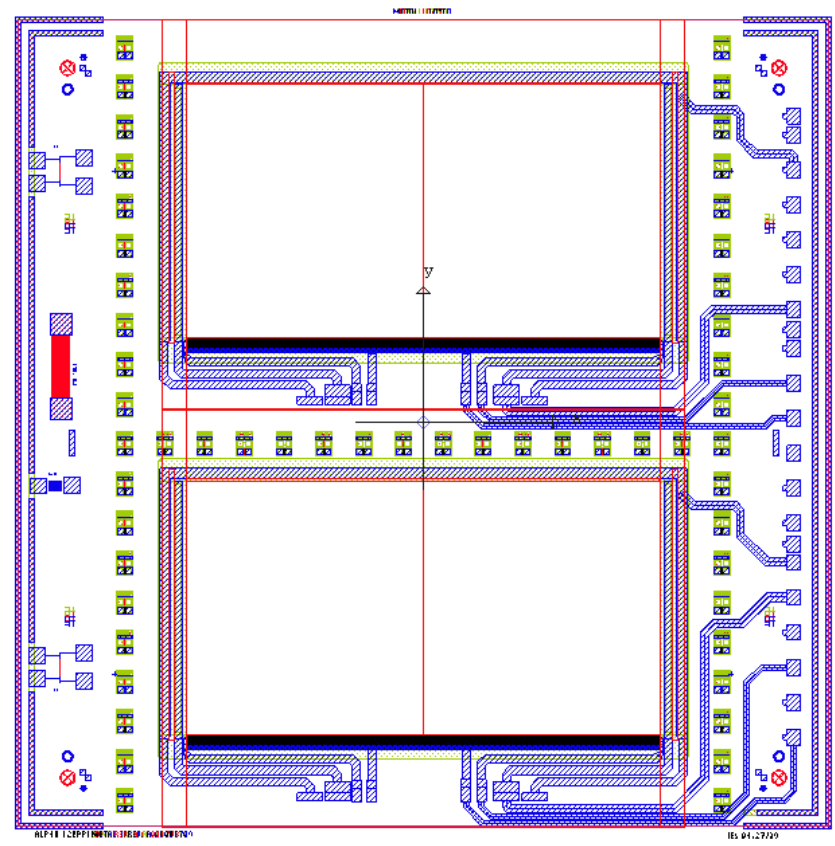

Fig.39. Schematic of the 6" square panels that are processed in DDI's pilot-line. There are two panels per substrate.

Instead of using an active matrix structure, a simpler (and cheaper) passive addressing system was chosen: every third colum of the display was 'ganged' together, i.e. all the blue colums were connected, as were all the orange columns and all the yellow columns. This simplified the electrical connections to four contacts: blue current, orange current, yellow current, and combined current return. (In practice the device is symmetrical with the contact pads repeated on both halves of the device - thereby giving redundency for the electrical connections). The 
blue columns were fed from one side of the panel (the 'bottom' in Fig.40), with the yellow and orange from the opposite edge (the yellow contacts had to pass through vias in an insulating layer in order to isolate them from the orange connections). The blue, orange and yellow contacts connect directly to bus-lines which extended through the emissive area of the display to feed the ITO contact of each pixel. The current return from the cathode was routed from both edges of the active area. All the current feed and return lines terminated on the 'top' edge of the panel to allow easy connection to external drive electronics, using 'tab-bonds'. This is shown schematically in Fig.40.

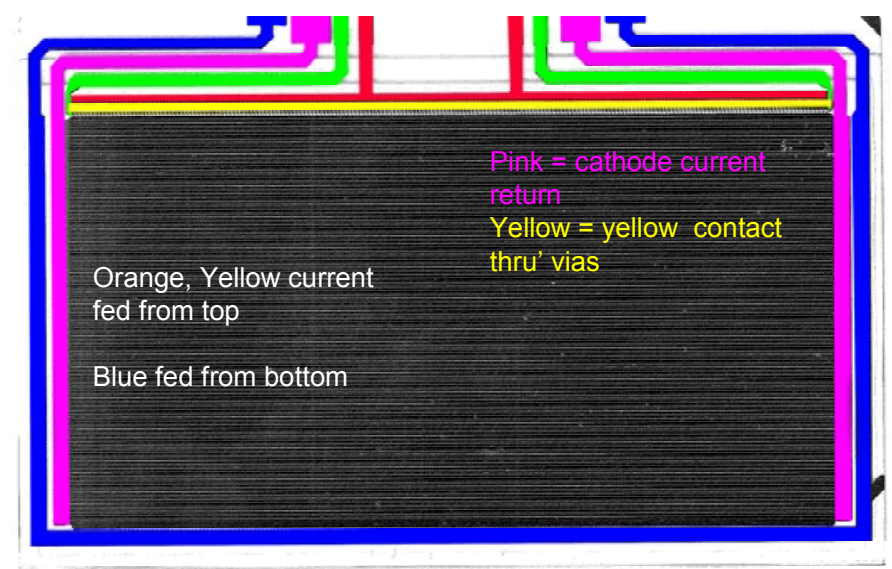

Fig.40. A more detailed schematic of a lighting panel showing the routing of the current bus lines.

\subsection{Thermal characterization of the Lighting Panel}

As well as the optical characteristics of the panel, it is important to consider its thermal properties: elevated temperatures will lead to reduced lifetimes and, in extreme case, panel failure. The problem boils down to one of how to extract the heat from the OLED panel and dissipate it into the atmosphere. The thermal properties of the various components were carefully considered and modeled, assuming a free-standing panel (i.e. not in a luminaire). Other input assumptions are shown in Fig.41.

- Modes of heat transport inside the device

Lateral heat transport:

1. Conduction along the electrical bus lines (high thermal conductivity path).



Transverse heat transport:

1. Conduction across the glass substrate

2. Conduction across cavity fill (gas or adhesive) + glass lid.

- Modes of heat transport out of the device:

1. Some conduction through the electrical leads (generally small contact area).

2. Convection from the front/back surfaces to the surrounding air.

3. Radiation from front/back surfaces (emissivity of glass $=0.94$; metals $=0.09$ ).

Fig.41. Heat transport mechanisms around and out of the package. 


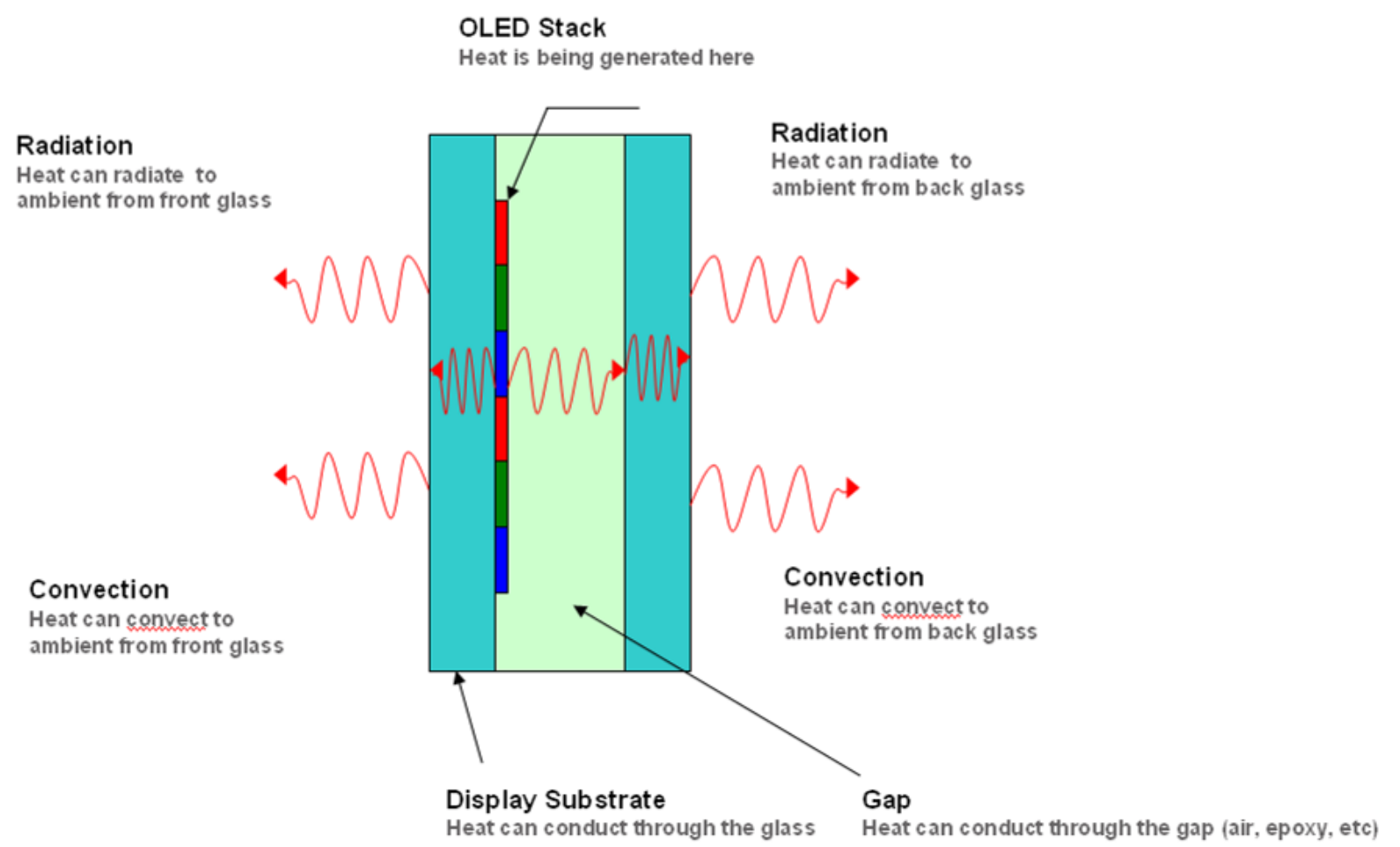

Fig.42. A simple 1-D model of our device: how can heat escape from the package?

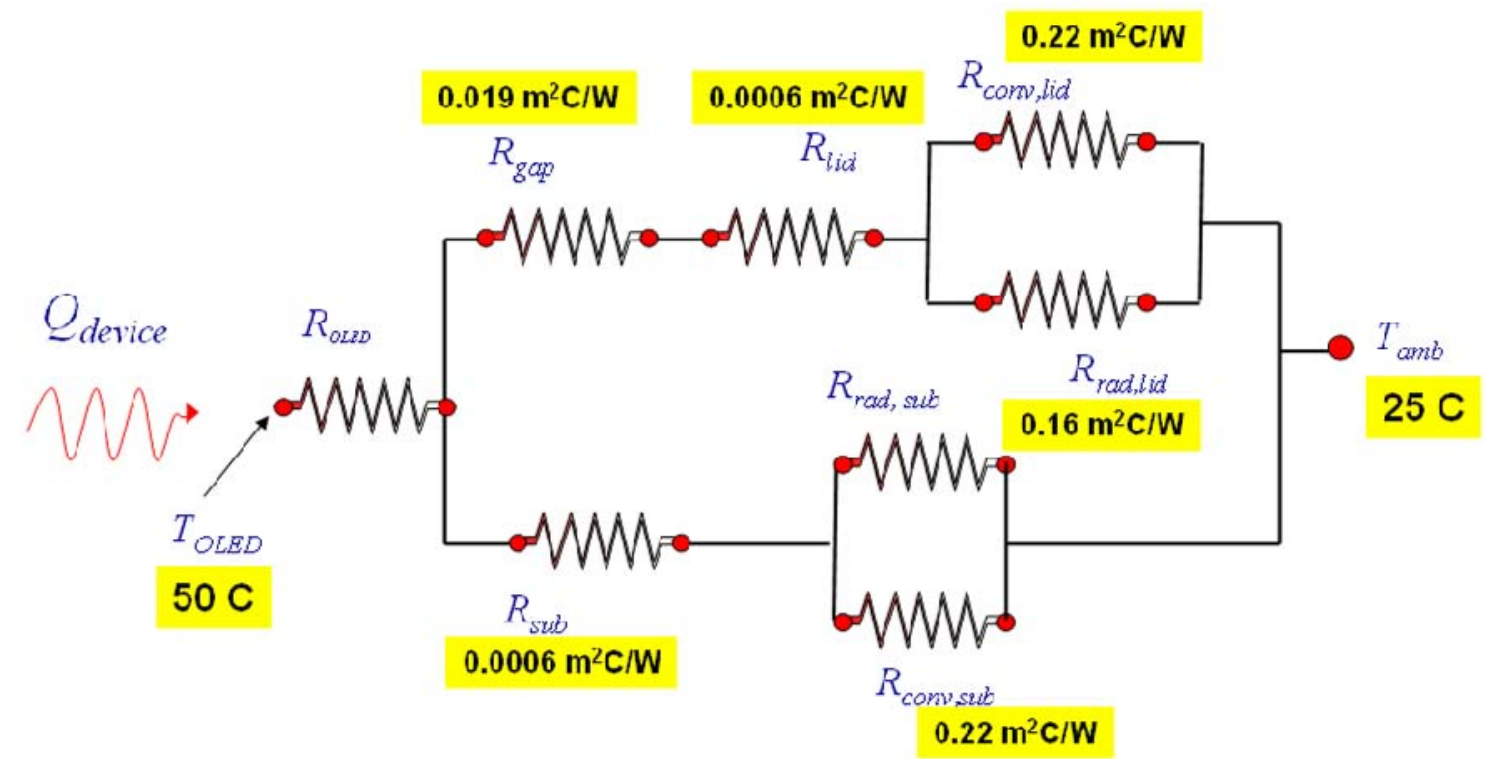

Conclusions:

1. Decreasing the Convection Thermal Resistance (Radiation torm already near minimum) will have the Greatest Affect on the Heat Transport out of the package.

2. Thermal Resistance of the Glass Plates and the "Gap" are Negligible.

Fig.43. Model for heat transport in our package, and estimates for the thermal resistivity terms. 
Our OLED panel includes a 50 micron 'air-gap' between the cathode and the encapsulating glass lid (actually $\mathrm{N}_{2}$ gas). Particular attention was given to understanding if this would be a thermal 'bottleneck' for heat dissipation. Figs. 42 and 43 show the model used to understand the heat flow.

The model shows that, in fact, the 'air-gap' is not a thermal bottleneck. The least effective thermal transport is by convective dissipation from the front and back of the panel (i.e. the highest thermal resistance). In other words, the operational temperature of a free-standing OLED lighting panel will be determined, almost entirely, by the degree of convective cooling.

Considering the limitations of convective cooling for a practical luminaire design, alternative cooling options were considered. For example, active cooling using a Peltier cooler was evaluated. Although very effective at lowering the panel temperature, the power requirements of the Peltier element are far too high. Simple calculations (shown below for a $400 \mathrm{~cm}^{2}$ lighting panel, drawing $5 \mathrm{~W}$ of electrical power) suggest the Peltier would have a power requirement similar to the panel itself, thereby lowering the overall efficacy of the system by $50 \%$ - an unacceptable tradeoff.



\section{Peltier Coolers}

Can we use a Peltier cooler to actively cool the OLED in order to extend its lifetime?

Yes - but at a substantial cost : the power required to drive the Peltier is comparable to that needed for the OLED (Peltiers are very inefficient).

The total power for the system might be increased by $\sim 50 \%$.

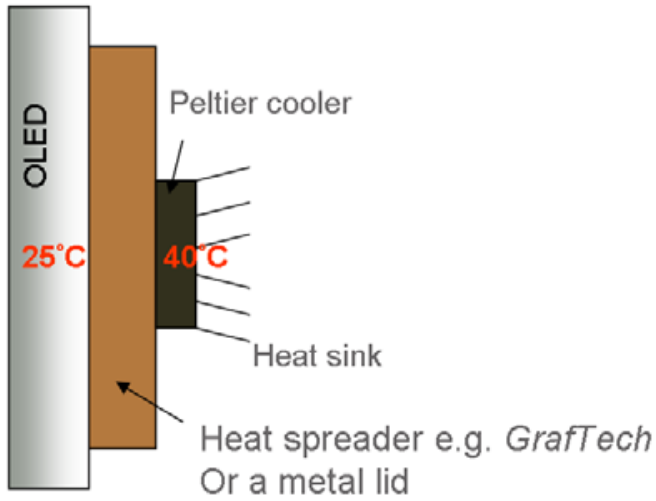

\section{Very rough calculation: \\ To keep OLED at $25^{\circ} \mathrm{C}$, with the heatsink @ $40^{\circ} \mathrm{C}$ ...and to pump $5 \mathrm{~W}$ of power from the OLED (so that it is in equilibrium) \\ Peltier requires $\sim 1.2 \mathrm{~A} @ 2 \mathrm{~V}$ i.e. $\sim 2.5 \mathrm{~W}$}

Fig.44. Simple calculations suggest that, although Peltier coolers would be very effective in keeping a panel cool, their poor efficiency lowers the overall system efficiency by too much for practical consideration. 


\subsection{First Generation Luminaire}

A simple packaging solution was chosen for the first generation luminaire design: the OLED panel was attached, using a high thermally-conductive material, to a thermally conductive chassis fabricated from aluminum. This allowed the heat to be distributed over the luminaire giving a larger area for convection and magnifying the cooling efficiency. GrafTech (GrafTech, USA, www.egraf.com) is specifically designed for such purposes and consists of a graphiteladen thin sheet with very high thermal conductivity (up to $1500 \mathrm{~W} / \mathrm{m} . \mathrm{K}$ - compared with $400 \mathrm{~W} / \mathrm{m} . \mathrm{K}$ for copper, or $200 \mathrm{~W} / \mathrm{m} . \mathrm{K}$ for aluminum).



Heat Transfer to Environment

Fig.45. Schematic of initial luminaire design. High thermal conductivity materials (e.g. GrafTech) can be used to move the heat to the outer surface of the luminaire to increase the surface area for convective cooling.

Fig.46 shows the initial luminaire design. The emissive area was $50 \mathrm{~cm}^{2}$, with a total thickness of $4.3 \mathrm{~mm}$. The inside of the aluminum chassis, adjacent to the edges of the OLED panel was covered with a layer of White $97^{T M}$ film (a $90 \%$ reflective, Teflon ${ }^{\circledR}$ based product from WhiteOptics, New Castle, DE, USA), in order to help scatter the edge-emission from the lighting panel. This added up to $5 \%$ to the light emission from the panel.
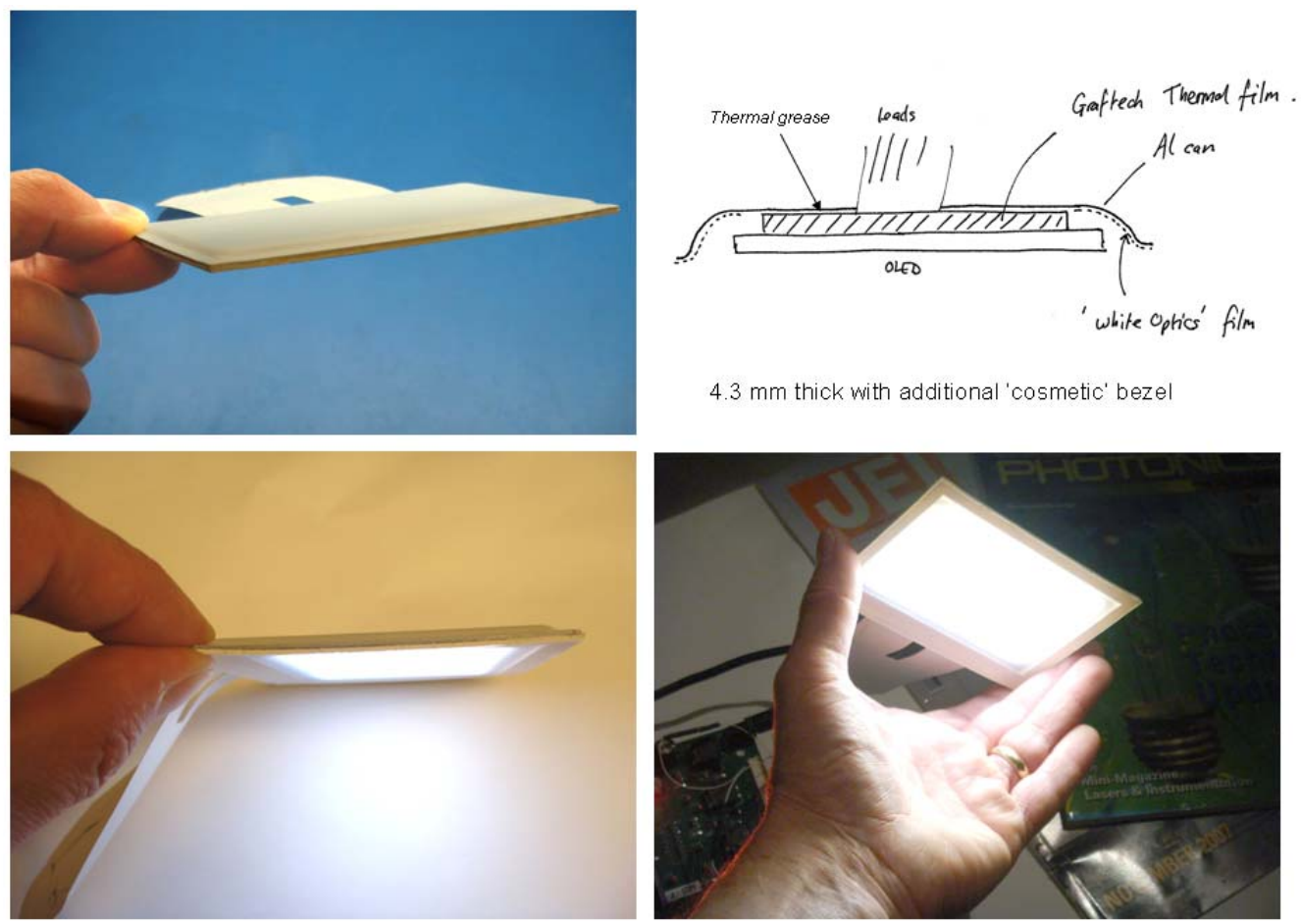

$4.3 \mathrm{~mm}$ thick with additional 'cosmetic' bezel

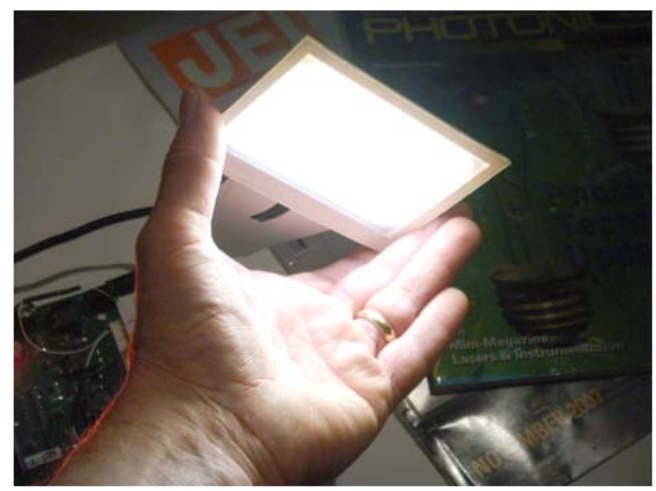

Fig.46. Initial Luminaire Design 


\subsection{Thermal Management and Panel Redesign}

Fig.47 shows the measured temperatures of panels mounted in these luminaires under likely operating conditions, i.e. in the horizontal orientation, and at luminance levels close to 2,000 nits (for whites). Figures represent the temperature, in ${ }^{\circ} \mathrm{C}$, measured in center and corners of the panel) for different colors.

In equilibrium, panel and luminaire temperatures typically operated $\sim 20^{\circ} \mathrm{C}$ above ambient, in still air. However, this was reduced to only $\sim 5^{\circ} \mathrm{C}$ if a small fan was used to increase circulation around the luminaire - verifying the model presented earlier regarding the limitations of cooling.

An increase in the operating temperature of the OLED by $20^{\circ} \mathrm{C}$ has significant impact on its operating lifetime - expected to reduce it by $\sim 4 \mathrm{x}$. This will be discussed in later sections. (N.B. this was substantially reduced in the second generation luminaire)

Fig.48 shows four of these luminaires mounted in light boxes at SID 2011 tradeshow. Two luminaires were fitted into each light box, each operating at $1500-2000 \mathrm{~cd} / \mathrm{m}^{2}$. Visitors could choose the color temperature: $2700 \mathrm{~K}$ white, $6500 \mathrm{~K}$ white, or one of the Orange-Yellow-Blue primaries. Due to the restricted convection in these enclosed boxes, small fans were used to circulate air around the devices to prevent overheating (the small circular openings above each panel).

- The panel temperature is limited by our ability to remove the heat.

- $\Delta \mathrm{T}$ was $\sim 10-20^{\circ} \mathrm{C}$ above ambient: this is expected reduce lifetime $\sim 4 \mathrm{x}$.

- A fan is very effective at removing heat from the panel and lowering its temperature - as expected from the model.
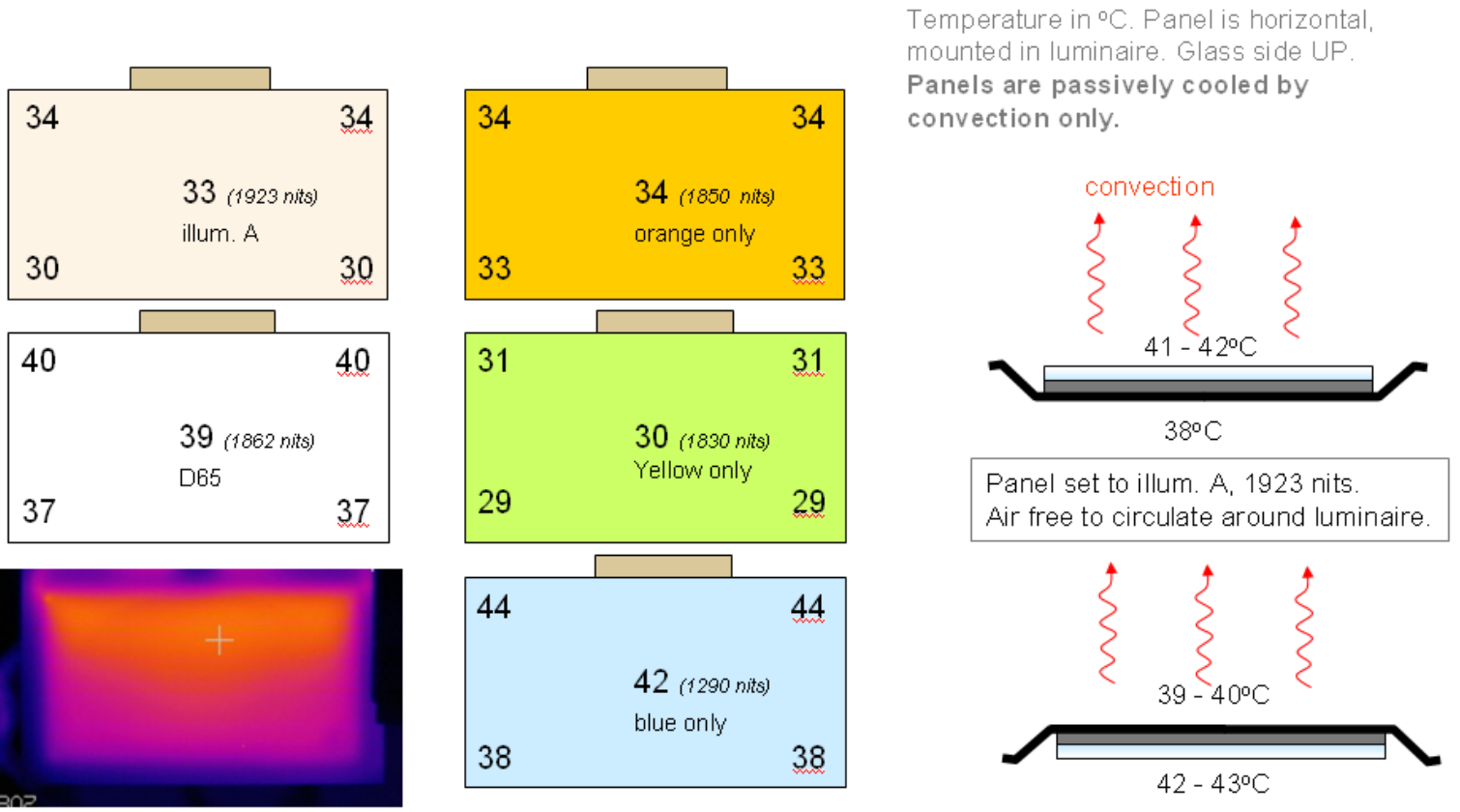

Fig.47. Temperature study of the luminaires. 


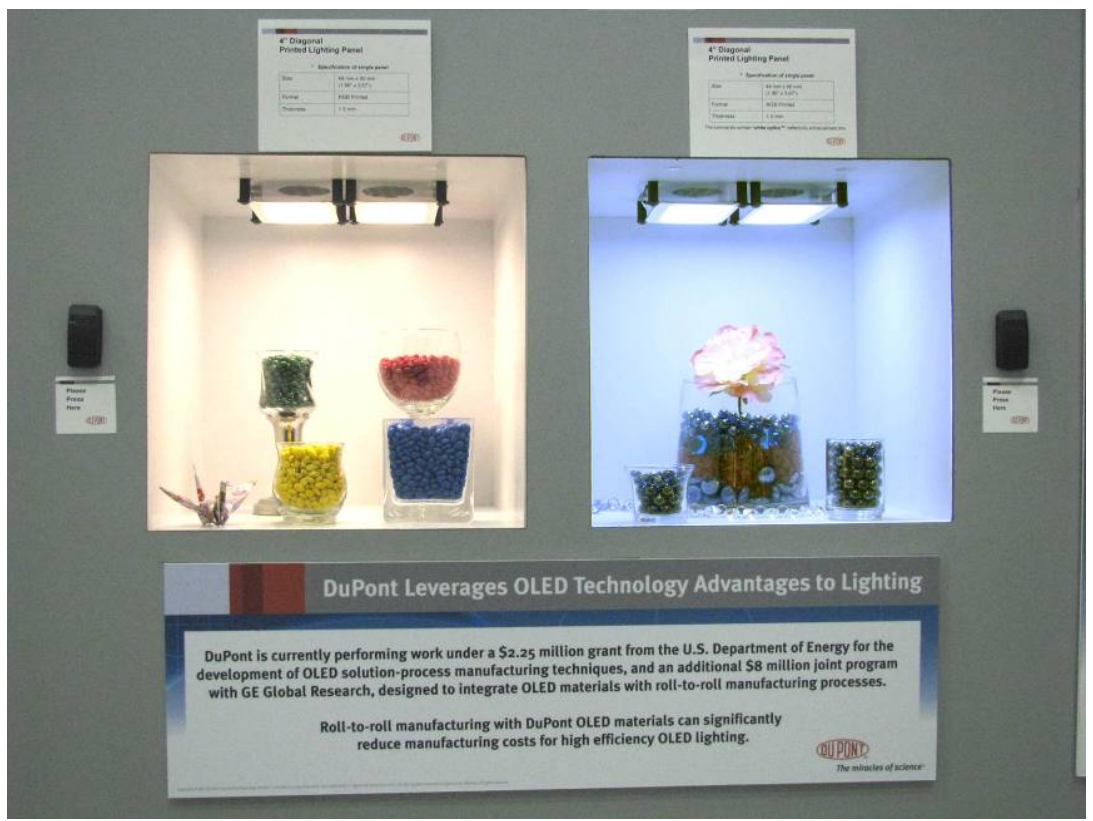

Fig.48. Devices mounted in light boxes at SID 2011 tradeshow. Two luminaires were fitted into each light box, both operating at 1500 - 2000 nits. Visitors could choose the color: $2700 \mathrm{~K}$ white, $6500 \mathrm{~K}$ white, or the Orange-Yellow-Blue primaries.

The first iteration of the panel design revealed some design problems, most notably the formation of hot-spots due to ohmic-heating of the current supply leads, and contact resistance of the tab-bond connections (see for example, Fig.49). The current supply leads were found to be too narrow (hence too resistive), and too closely spaced on the panel to allow effective heat dissipation. The tab-bonds used a pressure activated, silver-ball adhesive to make electrical contacts to the panel. This is a very successful technique for contacting display panels, but the current requirements for these lighting panels are considerably higher which resulted in repeated failure. Furthermore, the allocation of the blue current lead to the longest bus-line was problematic since the blue channel draws the highest current (it has the lowest efficacy) thereby exacerbating the ohmic heating problem.

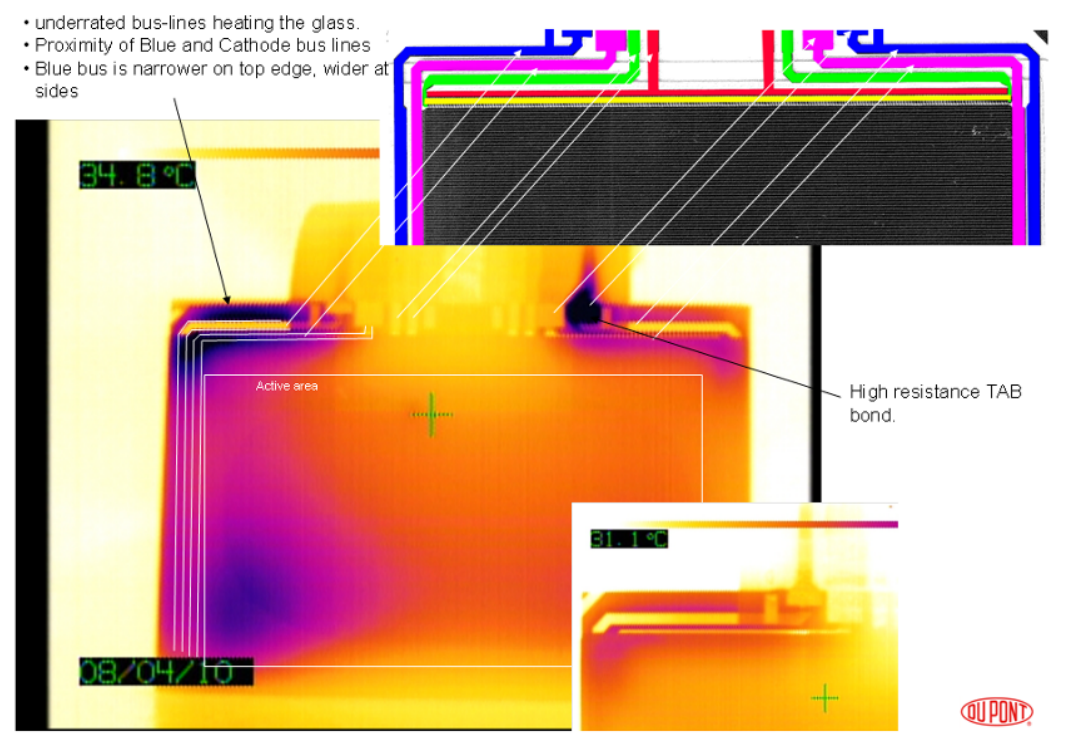

Fig.49. IR camera imaging of a panel. Only the blue channel is operating in this image. 
In order to rectify these problems, a finite-element model of the panel was developed (in conjunction with Blue Ridge Numerics, using the CF-Design tool). The heating problems associated with the original panel layout were replicated in the model and their origins confirmed. More importantly, an optimized panel layout was created. The current distribution lines were increased in width (as needed to accommodate their current capacity), the lines were re-ordered with the highest current blue-lines now feeding from the bottom edge - thereby minimizing their length, and contact areas were increased in size. Overall this led to lower current-densities, joule heating and voltage drop, as well as a more uniform distribution of heat across the panel. Fig.50 shows the modeled current density for the old and new designs.



Original layout
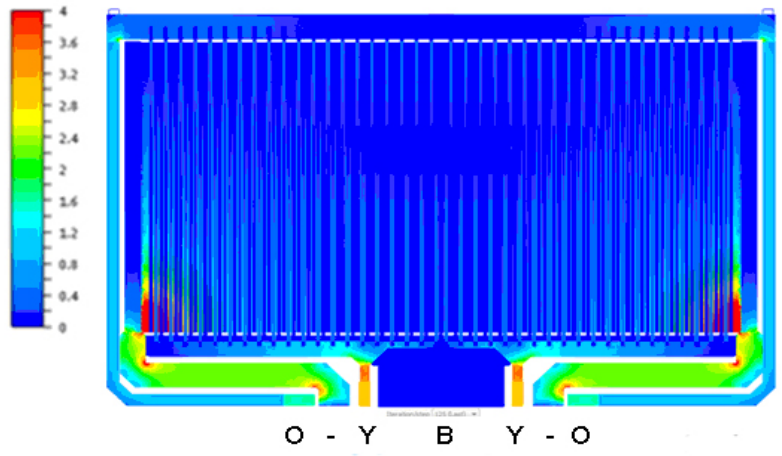

Redesigned layout

Fig.50. Results of the finite-element analysis for original and redesigned panel layout. Note the increased width of the current bus-lines, in particular the current return line (labeled " - "), with the commensurate reduction in current density and ohmic heating. Also note the reordering of the current distribution lines. In particular the blue line is now the shortest, a better choice since it carries the highest current of the three colors. 


\subsection{Drive Electronics}

The drive requirements for these color-tunable lighting panels are conceptually simple: three independent circuits are required (one for each color), which can be individually varied to change the ratio of the orange:yellow:blue luminance levels - thereby allowing continuous tuning of the overall panel color (and luminance) within the gamut defined by the three primary colors.

In practice this can be achieved in several ways, e.g. using a DC drive scheme, with either voltage or current sources, or by using a pulsed drive scheme - varying either the width or height of the pulses. There are pros and cons for each approach, but for manufacturing simplicity, low component cost and general flexibility it was decided to use a pulse-widthmodulation (PWM) drive scheme.

The driver circuitry was designed to have two controls - one potentiometer to adjust the color temperature of the OLED panel (32 steps along the black-body locus from illuminant-A to D65), and a second to adjust the overall luminance of the panel (255 steps from $\sim 0$ to $\sim 4,000 \mathrm{~cd} / \mathrm{m}^{2}$, $\sim 10,000 \mathrm{~lm} / \mathrm{m}^{2}$ ). This tunability was achieved by using a small microprocessor to maintain a 'look-up table' for the color ratios of the various whites, and to generate three appropriate PWM waveforms. High efficiency 'buck' LED-drivers, modulated by these PWM signals, provided constant current PWM waveforms for the three color channels.

Further considerations included how to step-down the $120 \mathrm{~V} \mathrm{AC}$ line voltage to the low-voltage requirements of typical circuitry (usually $5-12 \mathrm{VDC}$ ). It is important to achieve this with high efficiency in order to maintain the overall system efficacy. In practice it was decided (for generality) to use a 2-step process for this: a standard, high efficiency wall (switching) transformer to step down the $120 \mathrm{~V}$ line voltage to $12 \mathrm{VDC}$ to power the controller unit (typical efficiency is $\sim 85 \%$ ), followed by further step-down within the controller to power the various components and circuits. In practice this included a high-efficiency voltage regulator $(\sim 80 \%$ efficient) to step down to 5VDC for the microprocessor, and three separate buck drivers to provide constant current pulses for the three color channels ( $90 \%$ efficient).

Component count and price were considered, in order to keep total controller cost as low as possible. For example, a low cost microprocessor was selected (Atmel PIC16F1827, \$1.30 in small quantity) and a high-performance buck driver designed for LED applications (Allegro, A6210 - \$0.78 in small quantity). The remaining components are simple surface-mount resistors, capacitors, inductors, and appropriate connectors. The schematic for the controller circuit is shown in Fig.51.

The look-up table parameters were calculated based on the current-voltage-luminance data measured in the integrating sphere for completed panels, in combination with modeling software to calculate the ratio of the three colors needed for each 'value' of white. The PIC microprocessor firmware (code to read the color- and brightness-potentiometers, to reference the look-up table, and to generate the appropriate PWM outputs) was programmed in a standard fashion, using an EPROM programmer. 




Fig.51. Schematic for the controller electronics as described in the text. 


\subsection{Panel Performance with PWM Drive Scheme}

Due to the fact that the current was fed only from one edge of the panel, and due to the finite resistance of the bus-lines carrying current down the columns of the panel, some degree of luminance gradient was noted, with panels being brighter closer to the current contact. Furthermore, due to the asymmetry in feeding current for the different colors, it was found that care was needed to minimize color gradients across the panel.

Since the (instantaneous) currents are higher for the PWM drive, resistive losses are larger and the gradients more noticeable compared with a DC drive scheme. This is shown in Fig.52 for some preliminary device studies. However, optimization of the PWM drive scheme and the buck convertor circuit, to minimize the instantaneous currents, while still allowing for the full range of colors, was found to reduce this problem to barely noticeable levels. Still, future iterations of the panel design would benefit from a lower resistance bus-line to further reduce this problem.


Fig.52. Luminance and color gradients across the panel in DC and PWM-drive modes. The \% values indicate the difference from the center of the panel. The absolute luminance at the center of the panel is noted. After optimizing the output voltage level of the buck controllers, in combination with adjusting the PWM pulse widths, these gradients were significantly reduced. 


\section{7_ Second Generation Luminaire Design}

As a more advanced demonstration of an OLED lighting design, we developed a prototype task-light in collaboration with LITECONTROL (Hanson, MA). The OLED panels and electronics were fabricated by DuPont Displays, luminaire design and fabrication was by LITECONTROL.

This luminaire consisted of two OLED panels incorporated into a more sophisticated housing, as can be seen in Fig.53. The same control unit as previously described is used for this design, although now requiring two circuits per luminaire. This gave the following performance:

- Tunable color between illuminant-A and D65

- Variable emittance : from $0-10 \mathrm{k} / \mathrm{m} / \mathrm{m}^{2}\left(\sim 0-4 \mathrm{kcd} / \mathrm{m}^{2}\right)$

- Desktop illuminance levels : up to $45 \mathrm{ft} . c d$.

The luminaire was fabricated from $1 / 4$ " thick aluminum panels, powder-coated white, and proved to be an effective heat-sink by virtue of both the increased surface area (for convective dissipation) and by the bulk of metal available to absorb heat. When running at $10 \mathrm{klm} / \mathrm{m}^{2}$, the OLED panels were operating only $\sim 5^{\circ} \mathrm{C}$ above ambient temperature.
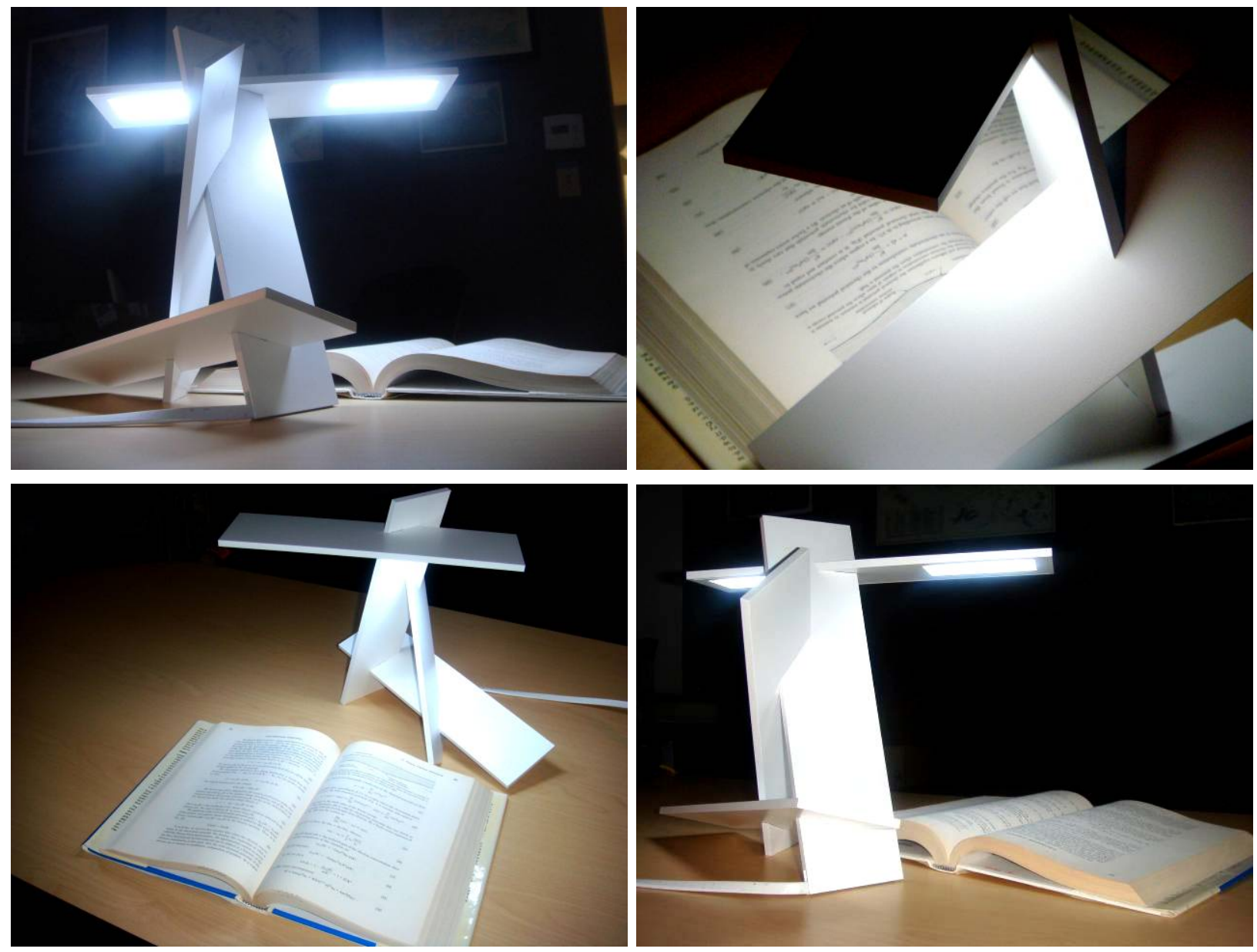

Fig.53. The second generation luminaires, designed in conjunction with LITECONTROL. 




Fig.54. Detail of the mounting of OLEDs into the luminaire. The cosmetic bezel is ready to be attached to cover all wiring.

\section{Manufacturing cost model}

The manufacturing cost-model is detailed and contains information that DDI considers to be proprietary. The full analysis is attached as a confidential Appendix. However, summarized results are included in section 11 - Summary. 


\section{Summary}

DDI is taking a novel approach to the fabrication of OLED solid-state lighting, using solution processed OLED technology. Organic layers are deposited by slot-coating and nozzle-printing techniques. The majority of process steps take place under atmospheric conditions rather than in high-vacuum. The aim of this project is to determine if this approach is feasible for the manufacture of low-cost, high performance and color-tunable lighting panels.

This report details the development work leading to the fabrication of $50 \mathrm{~cm}^{2}$ lighting panels, which were incorporated into task-lighting luminaires. These panels were spatially separated three-color devices, with separately printed colors. By controlling the ratio of these three colors, the color temperature of the overall emission color could be continuously adjusted - from a 'warm' illuminant-A ( 2700K), to a 'cool' D65 $(6500 \mathrm{~K})$. Panels reached a power efficacy of $35 \mathrm{Lm} / \mathrm{W}$ at illuminant-A (although it is anticipated that 42 $\mathrm{Lm} / \mathrm{W}$ would have been achieved with optimized ITO), while operating lifetimes easily met the $5,000 \mathrm{hr}$. target.

The materials selection and device architecture is reported in detail, along with specific decisions regarding the panel architecture aimed at providing a robust device. Engineering challenges are discussed - including thermal management issues and the drive electronics design. Panel performance is discussed in detail, including material efficacy $(\mathrm{Im} / \mathrm{W})$, lifetime, color-rendering values, and angular dispersion characteristics. Deficiencies in the performance of materials (notably blue) are reviewed.

One benefit of the laterally patterned device structure is that, since each color works independently of the others, upgrading one of the colors when a better choice becomes available (such as a more stable blue) is straightforward and doesn't require reoptimization of the whole device architecture, as would be the case for 'blended-color' or 'stacked' architectures. A further benefit of this architecture is that it is a trivial matter to rebalance the panel color if it should drift from the desired white-point - maybe as a result of differential aging of materials (this would not be possible for 'blended-color' architectures).

Finally a detailed cost-model is introduced. This model extends our current experience working with a Gen4 nozzle printer to a full-scale manufacturing line. This is a highly detailed model, for which a range of realistic scenarios are evaluated, meant to represent a realistic maturing of the technology over a period of years. The model predicts that large-scale solution-processing of OLED lighting panels is likely to be cost-effective, with module costs dropping to $\sim \$ 25$ per $\mathrm{kLm}$ and $\$ 25$ for a $1000 \mathrm{~cm}^{2}$ panel. (For the bare panels these numbers are roughly $\$ 18$ per $\mathrm{kLm}$ and $\$ 17$ per panel.) TAC time for nozzle printing will be 1 minute per Gen4 substrate for each printer. So a manufacturing line with a single nozzle printer can produce 220k lighting panels per month. Printing uniformity over Gen4 sized panels has proven to be excellent. Furthermore, the use of nozzle-printers provides significant flexibility in manufacture, since a change of material, or a change of device layout is easily accomplished through software, rather than mechanical tooling.

\section{Conclusions}

Large scale manufacture of OLED lighting panels using solution-based technology seems feasible and cost effective. Three-color lateral patterning of the panel is demonstrated to be a straightforward and flexible approach to color tunability. The device architecture required to achieve this is simple, and the drive electronics is low-cost.

The current materials set, however, demonstrates mixed performance: while the high efficiency materials show excellent lifetime and efficacy, the lack of a stable high efficiency blue material considerably lowers the overall panel performance. The necessitated use of a fluorescent blue reduced the overall efficacy to $35 \mathrm{Lm} / \mathrm{W}$ (potentially as high as $42 \mathrm{Lm} / \mathrm{W}$ ). Although some incremental improvements in the performance are anticipated with further optimization of the device architecture, substantial performance gains won't be achieved until a viable high efficiency blue is available. However, considerable effort is being devoted to the development of such materials, and it is anticipated that this limitation will be a temporary one. 